\title{
Tunable and Transferable Diamond Membranes for Integrated Quantum Technologies
}

\author{
Xinghan Guo, ${ }^{\dagger}$ Nazar Delegan, ${ }^{\dagger}, J^{\dagger}$ onathan C. Karsch, ${ }^{\dagger}$ Zixi Li, ${ }^{\dagger}$ Tianle \\ Liu, ${ }^{\mathbb{T}}$ Robert Shreiner, ${ }^{\mathbb{I}}$ Amy Butcher, ${ }^{\dagger}$ David D. Awschalom, ${ }^{\dagger}, \star, \mathbb{l}$ F. Joseph \\ Heremans, ${ }^{\dagger,+}$ and Alexander A. High ${ }^{*}, \dagger, \$$ \\ $\dagger$ Pritzker School of Molecular Engineering, University of Chicago, Chicago, USA \\ $\$$ Center for Molecular Engineering and Materials Science Division, Argonne National \\ Laboratory, Lemont, USA \\ IDepartment of Physics, University of Chicago, Chicago, USA \\ *E-mail: ahigh@uchicago.edu
}

\begin{abstract}
Color centers in diamond are widely explored as qubits in quantum technologies. However, challenges remain in the effective and efficient integration of these diamond-hosted qubits in device heterostructures. Here, nanoscale-thick uniform diamond membranes are synthesized via "smart-cut" and isotopically $\left({ }^{12} \mathrm{C}\right)$ purified overgrowth. These membranes have tunable thicknesses (demonstrated $50 \mathrm{~nm}$ to $250 \mathrm{~nm}$ ), are deterministically transferable, have bilaterally atomically flat surfaces $(R q \leq 0.3 \mathrm{~nm})$, and bulk-diamond-like crystallinity. Color centers are synthesized via both implantation and in-situ overgrowth incorporation. Within $110 \mathrm{~nm}$ thick membranes, individual germanium-vacancy $\left(\mathrm{GeV}^{-}\right)$centers exhibit stable photoluminescence at $5.4 \mathrm{~K}$ and average optical transition linewidths as low as $125 \mathrm{MHz}$. The room temperature spin coherence of individual nitrogen-vacancy $\left(\mathrm{NV}^{-}\right)$centers shows Ramsey spin dephasing times $\left(T_{2}^{*}\right)$ and Hahn echo times $\left(T_{2}\right)$ as long as $150 \mu \mathrm{s}$ and $400 \mu \mathrm{s}$, respectively. This platform enables the straightforward integration of diamond membranes that host coherent color centers into quantum technologies.
\end{abstract}




\section{Introduction}

Color centers in diamond are a leading platform in quantum networking and sensing due to their exceptional coherence times, ${ }^{1,2}$ robust spin-photon interfaces, ${ }^{3,4}$ and controllable interactions with local nuclear- and reporter-spin registers. ${ }^{5-7}$ Color centers have been utilized in many landmark experimental demonstrations, including deterministic entanglement, ${ }^{8}$ multi-node quantum networking, ${ }^{9}$ nanoscale NMR spectroscopy,${ }^{10}$ and memory-enhanced quantum key distribution. ${ }^{11}$ These advances can be accelerated into scalable technologies with the ability to create high quality, nanoscale-thick diamond membranes as elements in hybrid devices. Ideally, in the next generation of quantum technology, diamond will simply be another functional layer in heterostructures that can include non-linear/magnetic/acoustic materials, on-chip detectors, superconductors, nanophotonics, and microfluidics. Such improved integration can increase entanglement efficiency, ${ }^{9}$ master control of phonons and photons, ${ }^{11-13}$ enable on-chip frequency conversion, ${ }^{14}$ improve interfaces with other quantum systems and sensing targets, ${ }^{15,16}$ and create new opportunities to engineer quantum states. However, the material properties of diamond create fundamental difficulties for heterostructure integration. Specifically, high quality, single crystal heteroepitaxial growth of diamond thin films remains challenging despite recent progress. ${ }^{17,18}$ In response, a variety of processing and integration schemes have been developed to derive low dimensional structures out of bulk diamond. ${ }^{19-24}$ While promising, a scalable, high yield approach that enables full heterostructurelike integration of diamond while maintaining bulk-like properties - specifically, crystallinity, surface roughness, and color center coherence - is still lacking. Here, we report the efficient synthesis and manipulation of ultra-thin diamond membranes suitable for next-generation applications in quantum information science (QIS). Our membrane fabrication procedure is based on a "smart-cut" technique, ${ }^{23,25-28}$ in which $\mathrm{He}^{+}$implantation creates a subsurface graphitized layer that can be electrochemically etched, in conjuncture with a plasma enhanced chemical vapor deposition (PE-CVD) overgrowth optimized for the synthesis of high quality, in-situ doped, and isotopically purified diamond. The process allows detachment of arbitrarily large diamond membranes with smooth interfaces without relying on reactive- 
ionetching (RIE) undercut processes. While previous works have demonstrated the effectiveness of "smart-cut" and overgrowth for diamond membrane creation, our process realizes three critical advancements to the state-of-the-art. Firstly, we demonstrate isotopically controlled and nitrogen $\delta$-doped diamond membrane structures with atomically flat surfaces and high crystal quality showing unprecedentedly narrow Raman linewidths. ${ }^{28,29}$ Secondly, we demonstrate a novel drytransfer technique which enables clean and deterministic membrane placement on arbitrary substrates. Membranes transferred in this way are free from premature detachment and unwieldy curvatures caused by built-in strain between the graphitized and overgrown layers. ${ }^{30}$ As such, these membranes can be integrated with other material platforms and functional structures. Thirdly, we report that these structures, even at thickness $\leq 150 \mathrm{~nm}$, are suitable hosts for germanium vacancy $\left(\mathrm{GeV}^{-}\right)$and nitrogen vacancy $\left(\mathrm{NV}^{-}\right)$centers created via implantation (in both cases) and overgrowth $\left(\mathrm{NV}^{-}\right.$only). Specifically, we show that the $\mathrm{GeV}^{-}$centers exhibit stable and coherent emission at $5.4 \mathrm{~K}$, while the $\mathrm{NV}^{-}$centers show bulk-like spin coherence properties at roomtemperature. Additionally, we demonstrate that doping of other group IV color centers such as silicon-vacancy $\left(\mathrm{SiV}^{-}\right)$and tin-vacancy $\left(\mathrm{SnV}^{-}\right)$is also viable.

\section{Results and discussion}

\section{Diamond Membrane Synthesis}

The step-by-step fabrication procedure of the diamond membrane quantum platform is demonstrated in Figure 1a-e. The process starts with a low energy $(150 \mathrm{keV}) \mathrm{He}^{+}$implantation $\left(5 \times 10^{16} \mathrm{~cm}^{-2}\right)$ into diamond substrates, as shown in Figure 1a. This step forms a depth-localized graphitized underlayer $\approx 410 \mathrm{~nm}$ deep ${ }^{31}$ via damage-induced phase transition of the carbon bonds from $\mathrm{sp}^{3}$ to $\mathrm{sp}^{2}$. Unlike RIEbased undercut approaches, ${ }^{21,22}$ the top diamond layer maintains a uniform thickness and flatness throughout processing (see Figure S1b and Figure S3a in ${ }^{32}$ ). The substrates are then subjected to a multi-step anneal with a maximum temperature of $120{ }^{\circ} \mathrm{C}\left(\right.$ see section $1.1 \mathrm{in}^{32}$ ). The high temperature allows for the mobilization and subsequent annihilation of implantation-induced crystal damage in the 
top layer ${ }^{33,34}$ as characterized via Raman spectroscopy (see section 2.2 in $^{32}$ ). However, this process is imperfect, resulting in the top layer remaining unsuitable as a host for highly coherent color centers.

To fully mitigate the impact of implantation damage and achieve pristine crystal quality in the membranes, we follow the "smart-cut" with homoepitaxial PE-CVD of an isotopically engineered diamond thin film overlayer as shown in Figure 1b. During growth, the hydrogen to methane flow rate ratio is kept at $0.05 \%$ to ensure a morphology preserving step-flow growth regime. ${ }^{1,35}$ The growth rates herein were $6.2(4) \mathrm{nmh}^{-1}$ to $9.3(8) \mathrm{nmh}^{-1}$ for $700^{\circ} \mathrm{C}$ to $500^{\circ} \mathrm{C}$ heating plate temperature, respectively (see section $1.2 \mathrm{in}^{32}$ ). The process can be performed with higher growth rates to efficiently achieve thicker structures. In this work, we maintained low rates compared to other works ${ }^{23,26}$ to ensure a more accurate depth-localization of dopant layers, i.e., $\delta$-doping precision. ${ }^{1}$

We employed two distinct strategies for point defect creation within the overgrown layer. A subset of the membranes had a $\approx 2 \mathrm{~nm} \delta$-doped layer of ${ }^{15} \mathrm{~N}$ grown in, as schematized on the right of Figure 1c. These membranes underwent electron irradiation and subsequent annealing to form a $\delta$-doped $\mathrm{NV}^{-}$ layer (see section $1.2 \mathrm{in}^{32}$ ). Other overgrown diamonds were ion-implanted with $\mathrm{Ge}^{+}, \mathrm{Si}^{+}, \mathrm{Sn}^{+}$, and $\mathrm{N}^{+}$ as seen on the left of Figure 1c. These membranes were subjected to another identical multi-step anneal to form optically-active point defects via vacancy mobilization and annihilate implantation-induced crystal damage as much as possible. ${ }^{33,36}$

To realize a fully integrable diamond platform, we have engineered a high yield, controllable process to lithographically pattern membranes with desired shapes and sizes into the overgrown film, and subsequently transfer them individually onto other substrates/devices. The left of Figure 1f shows inductively coupled plasma (ICP)-defined square-shaped membrane arrays (200 $\mu \mathrm{m}$ side length) used in this work. Each step of the membrane definition and transfer utilizes established techniques and can be done in a cleanroom with standard equipment (see section 1.3 and $1.4 \mathrm{in}^{32}$ ). The size and shape of the membranes are fully defined and can be tailored to specific applications, with the maximum size only limited by the substrate dimensions. We fabricate membranes that are $200 \mu \mathrm{m}$ by $200 \mu \mathrm{m}$ as a demonstration of sufficient size for photonics integration. ${ }^{11,13,21}$ Manipulation of the membranes starts 
with an electrochemical (EC) etching of the graphitic underlayer as shown in Figure 1d. The substrate is placed in deionized (DI) water, with two electrodes in the vicinity $(\leq 25 \mu \mathrm{m})$ of the target membrane. These electrodes generate the EC potential necessary to selectively etch the graphitic layer(see section $\left.1.3 \mathrm{in}^{32}\right)$. Critically, in contrast to previous studies, ${ }^{26,30}$ a small portion of the underlayer is left unetched, creating a tether (selectively unetched diamond underlayer) that prevents premature membrane detachment before the dry transfer. The overall efficiency to transfer membranes onto a carrier wafer is greater than $80 \%$, mainly limited by human error during the EC etching. Therefore, from a single 3 $\mathrm{mm}$ by $3 \mathrm{~mm}$ diamond substrate, we can derive more than $45(200 \mu \mathrm{m} \times 200 \mu \mathrm{m})$ functional membranes.

Our transfer process, depicted in Figure 1e, draws inspiration from those utilized in van der Waals heterostructure fabrication. ${ }^{37}$ This process starts with a polydimethylsiloxane (PDMS) polycarbonate (PC) stack mounted on a micropositioner with angle and position precision of $0.001^{\circ}$ and $5 \mu \mathrm{m}$, respectively. This PDMS/PC stamp is used to uniformly dry-adhere and subsequently break-off the target membrane from the tether. The membrane is then flipped by introducing another PDMS stamp and placed on a hydrogen silsesquioxane (HSQ) - coated carrier wafer (see Figure S4 in ${ }^{32}$ ). Next, the structure is annealed at $600{ }^{\circ} \mathrm{C}$ to allow the HSQ to transition into a denser $\mathrm{SiO}_{\mathrm{x}}$ film. ${ }^{38} \mathrm{The} \mathrm{SiO}_{\mathrm{x}}$ film has a low thermal expansion ratio, ${ }^{39}$ low optical loss, ${ }^{13}$ is photochemically inert, and is compatible with most lithography schemes. The success of this work flow depends on the adhesion differences between PC, PDMS, and HSQ layers. This guarantees a transfer process that is highly deterministic, reproducible, and agnostic to carrier wafer and surface pattern choices. The overall height variation across the whole membrane is determined to be $\sigma \leq 10 \mathrm{~nm}$ (see Figure S5e in ${ }^{32}$ ). In this work, we bond diamond membranes to fused silica and thermal oxide wafers with pre-defined trenches, generating locally suspended regions. Suspension allows us to control the chemical termination of both surfaces and reduce HSQ-related fluorescence for photoluminescence (PL) characterizations of the embedded point defects(see section 1.8 and $2.4 \mathrm{in}^{32}$ for details). Finally, by flipping the membrane, we are able to fully etch-away the $\mathrm{He}^{+}$-damaged, graphitized diamond layer with chlorine-based ICP. This ICP etching eliminates the undesired fluorescence and built-in strain caused by crystallographic imperfections and lattice mismatch. ${ }^{27,32}$ The right part of Figure 1f shows a $20 \mathrm{~h}$ grown diamond 
membrane on a fused silica wafer with $100 \mathrm{~nm}$ final thickness. This etching step also serves to precisely tune the thickness of the final diamond structure(see section $1.4 \mathrm{in}^{32}$ ), with demonstrated thicknesses as low as $50 \mathrm{~nm} \cdot{ }^{13}$ As such, this membrane synthesis approach provides a clear, high yield, scalable, and easy path forward for generating multi-field relevant diamond membranes.
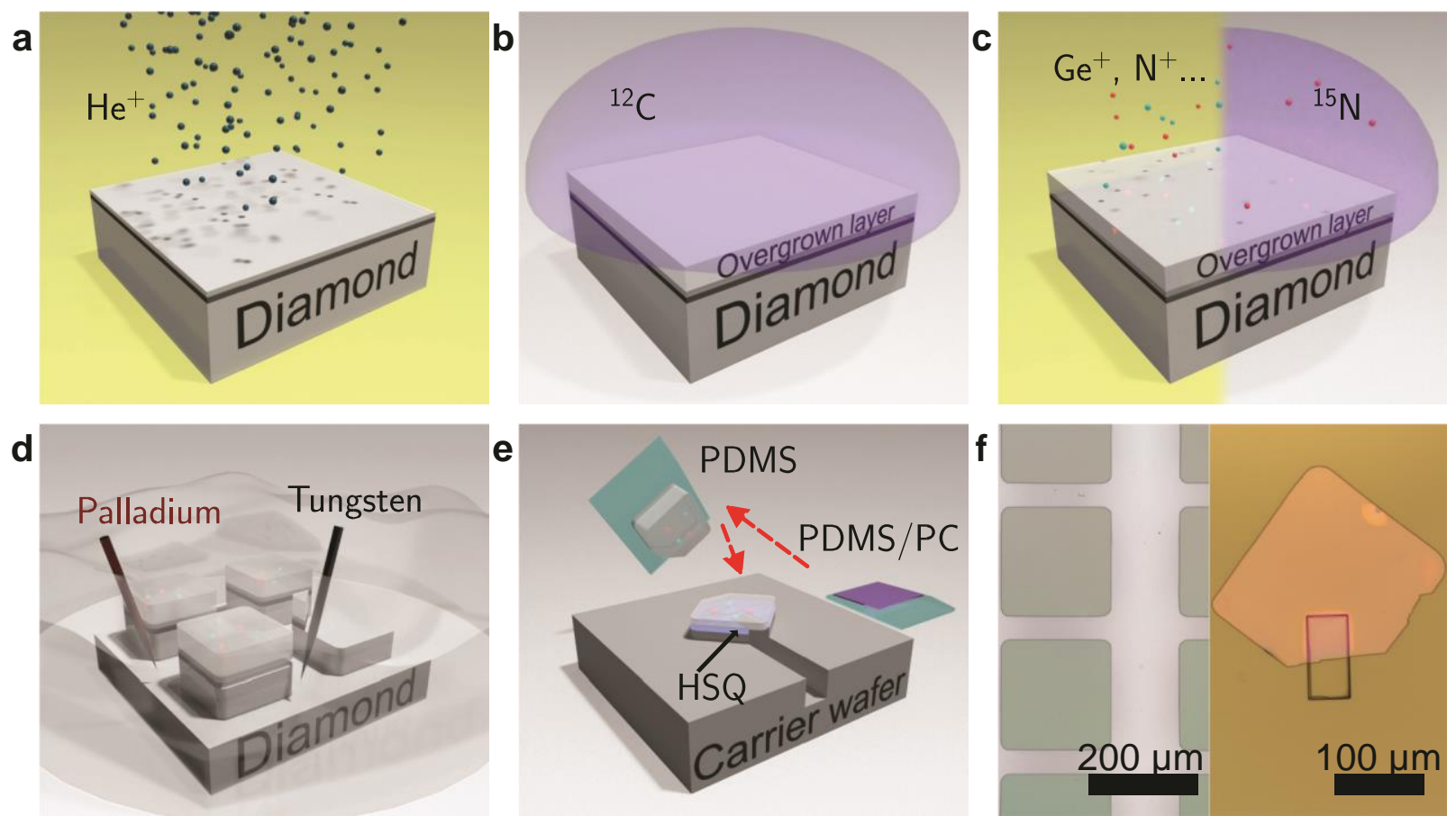

Figure 1: Schematics of the diamond membrane fabrication process. (a) $\mathrm{He}^{+}$implantation with subsequent annealing to form the membrane (light gray on the top) and the graphitized layer (dark grey underneath). (b) Isotopically $\left({ }^{12} \mathrm{C}\right)$ purified PE-CVD diamond overgrowth. (c) Color center incorporation via either ion implantation (left) or in-situ doping (right). Red dots: $\mathrm{N}^{+}$. Blue dots: $\mathrm{Ge}^{+}$. Other implanted species $\left(\mathrm{Si}^{+}, \mathrm{Sn}^{+}\right)$are not shown. (d) Diamond membrane undercut via EC etching. The undercut takes place in DI water, with palladium anode (red) and tungsten cathode (dark grey) aiming at the target membrane. (e) Membrane transfer and back etching. The membrane is picked up by the PDMS/PC stamp (green/purple), flipped onto another PDMS stamp (green), and bonded to the carrier wafer by HSQ resist (the blue layer sandwiched in between). (f) Microscope images of patterned overgrown membranes (left) and a transferred and multi-step etched membrane on a fused silica wafer (right). The green squares on the left are patterned membranes with underneath graphitized layer. 


\section{Surface and Material Quality}

The utility of diamond membranes in QIS demands exceptional surface and material quality. Figure 2 shows the atomic force microscopy (AFM) characterizations of the membrane backside (a-b) and topside (c) topology following all processing. We note that for both growth conditions $\left(500{ }^{\circ} \mathrm{C}\right.$ for $20 \mathrm{~h}$ and $700{ }^{\circ} \mathrm{C}$ for $40 \mathrm{~h}$ ), the resulting growth surface (i.e., the backside after dry transfer $)$ is smooth, showing distinct step-flow growth striations ${ }^{40}$ with a roughness $(\mathrm{Rq} \leq 0.31$ $\mathrm{nm})$ lower than the diamond lattice constant $(0.357 \mathrm{~nm})$. Similarly, the topside, although initially rough due to the intrinsic straggle of the $\mathrm{He}^{+}$implantation process (see Figure S1e in ${ }^{32}$ ), reaches $\mathrm{Rq}$ of $\leq 0.3 \mathrm{~nm}$ following ICP etching, ${ }^{41}$ as shown in Figure $2 \mathrm{c}$. The realization of atomically flat surfaces is critical for effective surface termination and coherence protection of near-surface color centers. $^{33}$

Additionally, the diamond crystal quality was investigated via room temperature Raman spectroscopy as shown in Figure 2d. To benchmark the spectra, we compare our values to a surface strain-released ${ }^{36}$ reference diamond (single-crystal, electronic (EL) grade) from Element Six, with a Raman linewidth of $1.570(1) \mathrm{cm}^{-1}$. The $\approx 185 \mathrm{~nm}$ overgrowth membrane $\left(20 \mathrm{~h}\right.$ growth in $500{ }^{\circ} \mathrm{C}$, back-etched down to $100 \mathrm{~nm}$ ) presents a Raman linewidth of $1.779(5) \mathrm{cm}^{-1}$, slightly larger than the reference value. Remarkably, the isotopically purified, $\approx 370 \mathrm{~nm}$ overgrowth membrane (40 $\mathrm{h}$ growth in $500{ }^{\circ} \mathrm{C}$, back-etched down to $110 \mathrm{~nm}$ ) presents a linewidth of $1.375(2) \mathrm{cm}^{-1}(1.486(14)$ $\mathrm{cm}^{-1}$ for the $\approx 250 \mathrm{~nm}$ isotopically purified overgrowth at $700{ }^{\circ} \mathrm{C}$, see Figure $\mathrm{S} 2 \mathrm{~b} \mathrm{in}^{32}$ ), significantly lower than anything reported previously, ${ }^{28}$ even compared to pristine natural isotopic abundance bulk diamond. The ultra-narrow Raman peak indicates the crystal is free of impurities and defects. The up-shifting and narrowing, in comparison with the bulk spectra, is consistent with the change of the Raman transition brought on via isotopic purification of a high-quality diamond structure. ${ }^{29}$ As such, the Raman spectra indicate that these diamond membranes are of ideal crystal quality to host color centers. 

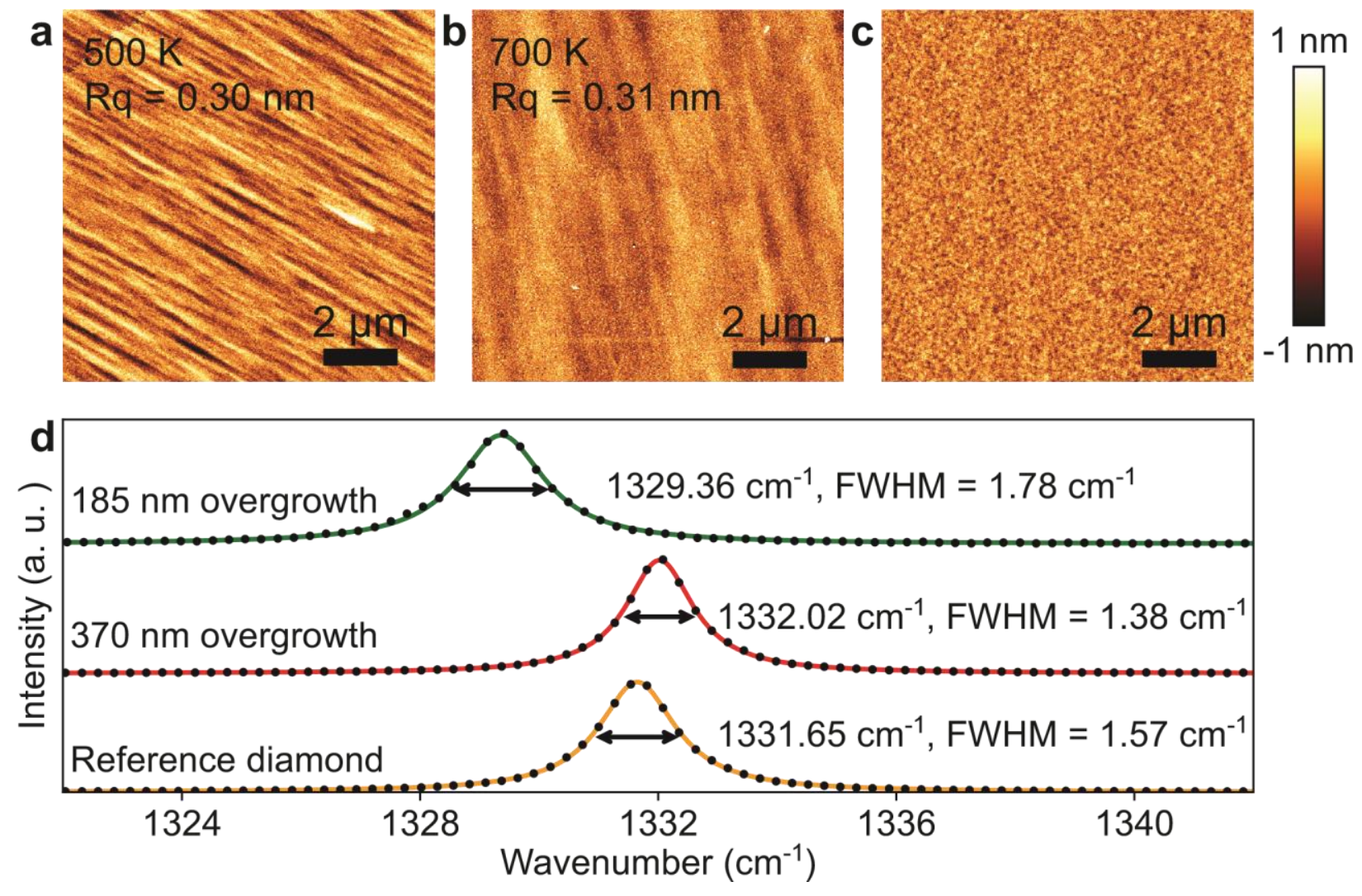

Figure 2: Surface morphology and crystal quality of overgrown membranes. (a-b) AFM images of overgrowth patterns (back side of membranes after the transfer) at different heating plate temperatures. (c) An AFM image on the front side of the membrane after multi-step etching. (d) Room temperature Raman spectroscopy of diamond membranes and the reference diamond substrate. Green: The $\approx 185 \mathrm{~nm}(20 \mathrm{~h})$ overgrowth membrane back-etched down to $100 \mathrm{~nm}$. Red: The $\approx 370 \mathrm{~nm}(40 \mathrm{~h})$ overgrowth, isotopically purified membrane back-etched down to $110 \mathrm{~nm}$. Yellow: A surface strain-released, EL grade single crystal diamond used as the reference. The surface strain is polish-induced, and is removed by ICP etching and subsequent annealing prior to the Raman spectroscopy(see section $2.2 \mathrm{in}^{32}$ ).

\section{Implanted Group IV Color Centers}

In order to investigate the coherence of color centers in the membranes, we performed detailed measurements of the optical (spin) coherence of $\mathrm{GeV}^{-}\left(\mathrm{NV}^{-}\right)$. We mounted the $110 \mathrm{~nm}$ thick ( $\approx 370 \mathrm{~nm}$ overgrowth), ${ }^{12} \mathrm{C}$-membrane on a trenched thermal oxide wafer with a vapor HF-based HSQ removal (see section $2.4 \mathrm{in}^{32}$ for details), as shown in Figure 3a. This HSQ removal is optional, and the additional HSQ fluorescence has minimal impact on device performance for $\mathrm{GeV}^{-}$resonant 
experiments. $\mathrm{GeV}^{-}$centers inside the sample were subsequently characterized at $5.4 \mathrm{~K}$. This particular membrane was also implanted with nitrogen (see further in the text), silicon, and tin (see section $\left.1.5 \mathrm{in}^{32}\right)$.

A PL map of the $\mathrm{GeV}^{-}$centers over the suspended area is shown in Figure $3 \mathrm{~b}$. The $\mathrm{GeV}^{-}$ conversion efficiency was determined to be $6.5(4) \%$ (see section $3.2 \mathrm{in}^{32}$ ). Typical zero phonon line (ZPL) peaks of $\mathrm{GeV}^{-}$centers lie between values reported in bulk diamond, ${ }^{42,43}$ indicating a homogeneous crystal environment (see Figure S6a $\mathrm{in}^{32}$ ). The span of the ZPL peaks across different centers come from the nature of the implantation. ${ }^{44}$ The inhomogeneous broadening of the ZPL positions are comparable with those measured in bulk diamond under identical implantation and annealing conditions. While strain generated from the membrane mounting, thermal expansion ratio mismatch with the carrier wafer, and HSQ annealing may potentially contribute to energetic variation, our measurements indicate the crystal environments between the diamond membrane and bulk diamond are highly similar (see section $3.5 \mathrm{in}^{32}$ ). Both off- and onresonance autocorrelation measurements were performed on the $\mathrm{GeV}^{-}$centers (see section $3.3 \mathrm{in}^{32}$ ), confirming anti-bunching features associated with single photon emitters with $g^{(2)}(0)=0.17(2)$ and 0.19(5), respectively. Additionally, the transition linewidths of the $\mathrm{GeV}^{-}$centers are relatively low, with single scan lines as narrow as 70(1) MHz. Furthermore, this optical transition is stable, offering a $2 \mathrm{~h}$ average spectral broadening as low as $124.8(2) \mathrm{MHz}$, as seen in Figure 3c. This broadening is still less than a five-fold increase over the intrinsic linewidth. ${ }^{42}$

For completeness, we also analyzed the statistical distribution of single-scan linewidths and time-averaged broadening. Out of 38 centers observed with off-resonant excitation, we were able to resolve the $\mathrm{C}$ transition in 29 centers with resonant scanning. Additionally, in 7 (1) centers, we observed the $\mathrm{C}$ transition switches between 2 (4) stable peaks (see section 3.4 in $^{32}$ for the 2 peaks pattern). The origin of this switching between two stable energies, distinct from random spectral diffusion, is the subject of ongoing study. Statistics of the other 21 single-peak $\mathrm{GeV}^{-}$centers yield a median single (2.5 min average) scan linewidth of $95 \mathrm{MHz}(231 \mathrm{MHz})$, as shown in Figure 3d. Recent cavity quantum-electrodynamics measurements ${ }^{45}$ have realized a spin-photon coupling rate 
of $g=2 \pi \times 5.6 \mathrm{GHz}$, significantly larger than the observed spectral broadening in our centers. Therefore, the membrane-hosted $\mathrm{GeV}^{-}$centers are sufficiently optically coherent for advanced applications in quantum networking and entanglement generation. These diamond membranes are also viable hosts for $\mathrm{SiV}^{-}$and $\mathrm{SnV}^{-}$centers (see section $3.3 \mathrm{in}^{32}$ ).
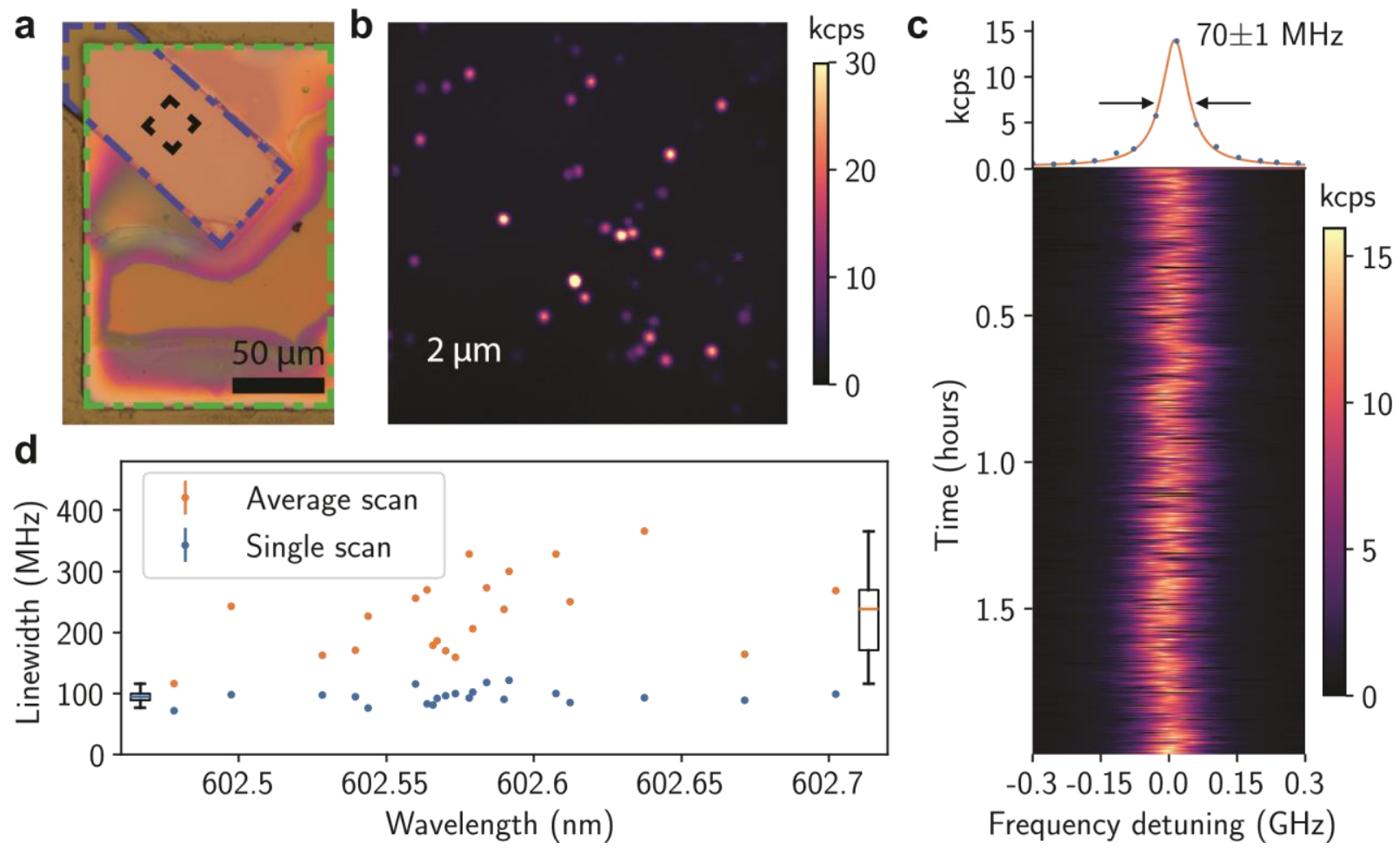

Figure 3: Optical characterization of $\mathrm{GeV}^{-}$centers at $5.4 \mathrm{~K}$. (a) A microscope image of a diamond membrane (dashed green) containing implanted $\mathrm{GeV}^{-}$centers. HSQ in the trench (dashed blue) is completely removed by vapor HF. The rainbow color on the membrane indicates excess HF undercut (see section $2.4 \mathrm{in}^{32}$ ). The PL measurement is performed in the dashed black region. (b) A PL map of the implanted $\mathrm{GeV}^{-}$centers. The signal-to-background ratio for most centers are between 5 to 30. (c) Single PLE scan and time-averaged broadening of a GeV center. The data sampling time per point is $100 \mathrm{~ms}$, and the counts are normalized to kilo-counts-per-second (kcps). (d) Statistics of $\mathrm{GeV}^{-}$optical linewidths measured via single-scan (blue) and average broadening (orange). The inset box plot on the left (right) indicates the median single (average) linewidth of $95 \mathrm{MHz}(231 \mathrm{MHz})$. 


\section{Embedded NV ${ }^{-}$Centers}

Next, we investigated the spin coherence of $\mathrm{NV}^{-}$centers in the membranes using a home-built room temperature PL microscope (see section $4.1 \mathrm{in}^{32}$ ). While the Zeeman splitting frequencies of group IV diamond color centers are one to two orders of magnitude larger than those of the $\mathrm{NV}^{-}$ center, ${ }^{2,46,47} \mathrm{NV}^{-}$coherence measurements reveal magnetic noise levels which are also relevant to group IV coherent control. ${ }^{33,48-50} \mathrm{All} \mathrm{NV}^{-}$center measurements presented herein were performed with a 15 gauss static magnetic field applied at $10^{\circ}$ angle to the [111] crystal axis. We measured $\mathrm{NV}^{-}$centers along all four possible crystal orientations, determined by different transition frequencies.

Figure 4a-b show representative free induction decay and spin echo decay curves on a single longlived $\mathrm{NV}^{-}$spin, with the fitted $T_{2}^{*}$ and $T_{2}$ coherence times. The oscillations in the first $100 \mu \mathrm{s}$ of Figure $4 \mathrm{~b}$ arise from aliasing of electron spin echo envelope modulation (a finer trace to demonstrate the origin of this is presented in the Figure S7c of ${ }^{32}$ ). Together, these measurements demonstrate that membrane fabrication does not preclude the formation of highly coherent spin qubits. Figure $4 \mathrm{c}$ presents a scatter plot of the $T_{2}^{*}$ and $T_{2}$ times, showing a spread of $4.3(3) \mu$ s to $149(7) \mu \mathrm{s}$ and $8(2) \mu \mathrm{s}$ to $400(100) \mu \mathrm{s}$, with most times (median 50\%) falling above $10 \mu \mathrm{s}$ and 30 $\mu \mathrm{s}$, respectively.

A common source of magnetic noise for $\mathrm{NV}^{-}$centers is unconverted nitrogen atoms ( $\mathrm{P}_{1}$ centers). Taking into account nitrogen originating from both implantation and background in-grown sources, we estimate a density $[N]$ of $\leq 1 \mathrm{ppb}$ (see section $4.5 \mathrm{in}^{32}$ ). At these concentrations, $\mathrm{P}_{1} \mathrm{~s}$ should not contribute heavily to the decoherence. ${ }^{51}$ Comparing the background nitrogen concentration and the observed areal $\mathrm{NV}^{-}$density versus the $[N]$ implantation dose of $2 \times 10^{8} \mathrm{~cm}^{-2}$, we expect that many of our observed $\mathrm{NV}^{-}$formed from in-grown nitrogen and vacancies introduced during ion implantation and not exclusively from the implanted nitrogen. Thus, the observed $\mathrm{NV}^{-}$are likely distributed throughout the thickness of the membrane, with some residing within $\leq 15 \mathrm{~nm}$ of both surfaces, where previous work demonstrated marked decoherence from surface noise. ${ }^{49}$ This 
ambiguity can be avoided by implanting ${ }^{15} \mathrm{~N}$ in future iterations of this experiment. However, statistically, the surface proximity distribution of the $\mathrm{NV}^{-}$alone cannot fully account for the large number of NV centers with $T_{2} \leq 100 \mu \mathrm{s}$. The multi-species implantation process is known to introduce crystal damage throughout the ion path, which can create spin-full vacancy complexes that are not mobilized, nor annihilated during the annealing process. ${ }^{50}$ It is likely that the resulting inhomogeneity of the bulk spin bath is the main factor limiting $\mathrm{NV}^{-}$coherence times. Nonetheless, the spin echo coherence time ( $T_{2}$ up to $400 \mu \mathrm{s}$ ) is competitive with near bulk-like properties, and the free induction decay ( $T_{2}^{*}$ up to $150 \mu \mathrm{s}$ ) outperforms commercially available bulk material due to the ${ }^{12} \mathrm{C}$ purification. Therefore, the coherence times presented herein are fully compatible with applications in quantum sensing and hybrid quantum systems. ${ }^{48,52,53}$

Additionally, we utilize $\mathrm{NV}^{-}$optically detected magnetic resonance (ODMR) measurements to characterize strain in the diamond membrane, which may be introduced by the fabrication. ODMR measurements at zero static field reveal splittings between upper and lower $\mathrm{NV}^{-}$transitions that are generally larger than shifts in the zero field splitting. We thus attribute these effects mainly to local electric field variations (see section $4.3 \mathrm{in}^{32}$ ). ${ }^{54}$ If we take the $1 \mathrm{MHz}$ observed shifts as an upper bound on strain, we find a maximum strain of $10^{-4} .55$

This membrane fabrication technique is also highly amenable to in-situ $\delta$-doping of ${ }^{15} \mathrm{~N}$ during overgrowth. ${ }^{1,32} \delta$-doping allows deterministic incorporation of dopants (N, Ge, Si, etc.) during membrane overgrowth while providing a valuable distinction from the intrinsic, isotopically naturally abundant defects (i.e., isotopically incorporating ${ }^{15} \mathrm{~N}$ during growth to distinguish from the ${ }^{14} \mathrm{~N}$ overgrowth background). As a proof of concept we introduced $2 \mathrm{~nm}$-thick area of ${ }^{15} \mathrm{~N}$ doping $\approx 36 \mathrm{~nm}$ from the backside of a $110 \mathrm{~nm}$ thick diamond membrane $(\approx 250 \mathrm{~nm}$ overgrowth at $700{ }^{\circ} \mathrm{C}$ ). Figure $4 \mathrm{~d}$ shows a PL map of NV centers in such a sample. The ${ }^{15} \mathrm{NV}^{-}$centers are labeled in teal circles, while background ${ }^{14} \mathrm{NV}^{-}$are in white rectangles, with representative hyperfineresolved ODMR spectra presented to the right of the figure. We observed a $7: 11$ ratio of $\left[{ }^{15} \mathrm{NV}^{-}\right]$: $\left[{ }^{14} \mathrm{NV}^{-}\right]$(SIMS characterized $\left[{ }^{15} \mathrm{NV}^{-}\right]$of $30.8(57) \mathrm{ppb}$, see section $4.5 \mathrm{in}^{32}$ ). This is in a good 
alignment with what was observed for the implantation-synthesised $\mathrm{NV}^{-}$centers, showing a consistent background ${ }^{14} \mathrm{NV}^{-}$density throughout the membrane from the overgrowth process. A rigorous quantitative comparison of optimized implanted and in-gown defects as they relate to the membrane surfaces proximity is left for subsequent studies to explore.

$\mathbf{a}$

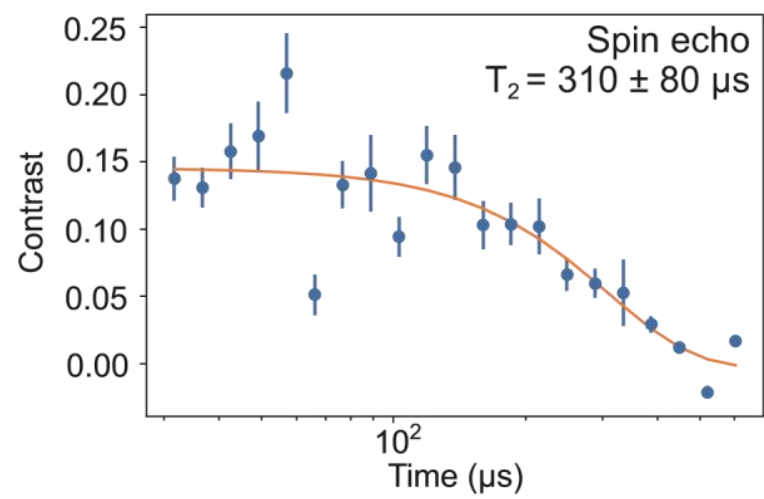

C

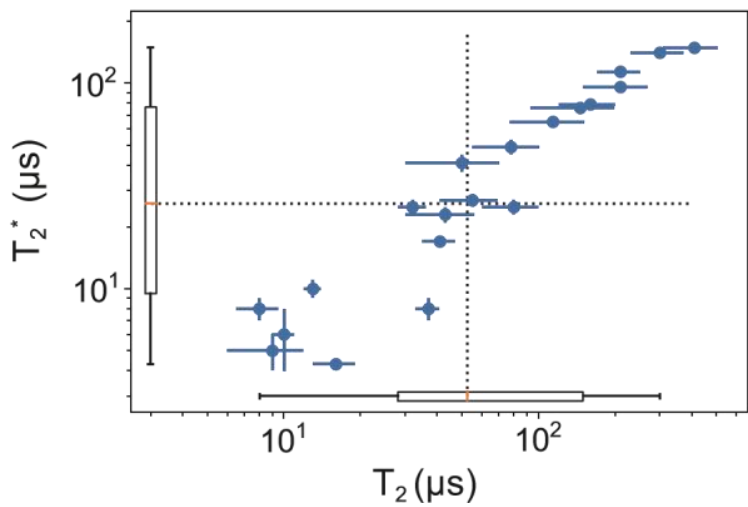

b

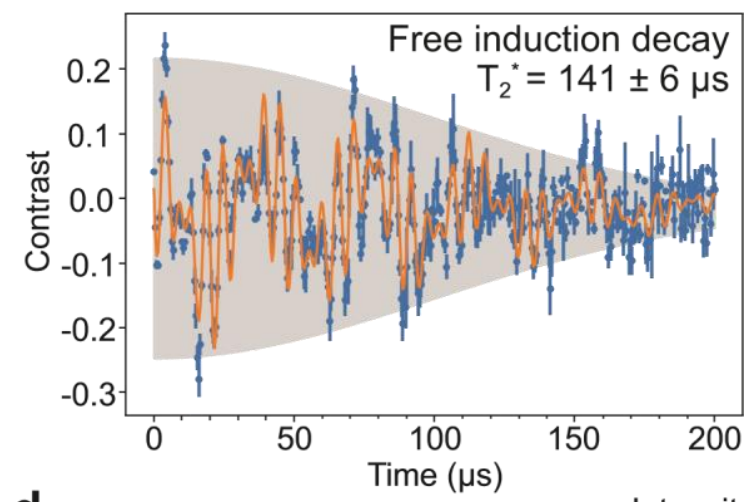

d
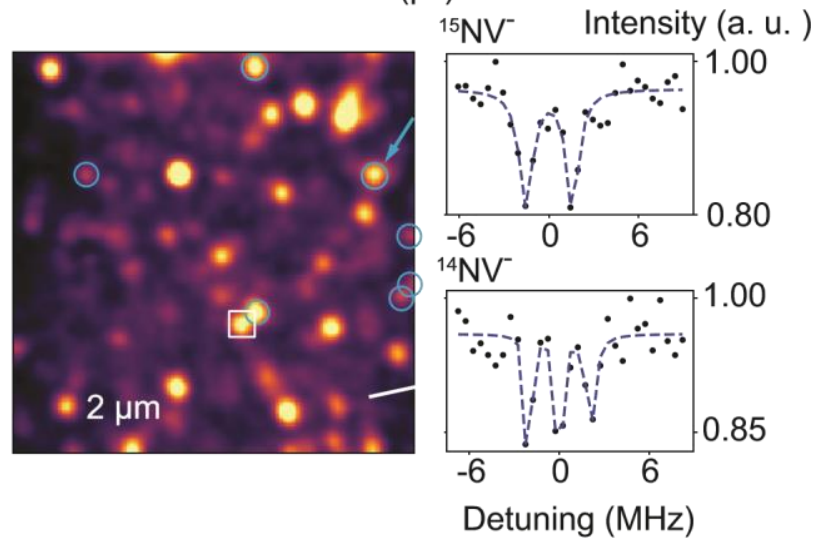

Figure 4: Optical characterization of embedded $\mathrm{NV}^{-}$centers at room temperature. (a-b) Representative free induction decay and spin echo decay curves on a single long-lived $\mathrm{NV}^{-}$spin, accompanied by the $T_{2}^{*}$ and $T_{2}$ coherence times. A detailed description of data analysis is provided in the Supporting Information. The oscillations in the first $100 \mu \mathrm{s}$ of (b) arise from aliasing of electron spin echo envelope modulation. (c) A scatter plot of $T_{2}^{*}$ and $T_{2}$ times for the 20 measured $\mathrm{NV}^{-}$centers. Inset box plots denote median values of $26 \mu \mathrm{s}$ and $52.5 \mu \mathrm{s}$ (dashed lines) and lowerquartile values of $9.5 \mu \mathrm{s}$ and $28 \mu \mathrm{s}$. Error bars are fit errors. (d) $\mathrm{NV}^{-} \mathrm{PL}$ map of a $\delta$-doped membrane with ${ }^{15} \mathrm{NV}^{-}$centers (teal circles) and ${ }^{14} \mathrm{NV}^{-}$centers (white squares) labeled. At right, pulsed-ODMR spectra of the indicated $\mathrm{NV}^{-}$centers. 


\section{Conclusion}

We have created uniform, tunable, transferable, large-scale diamond membranes with material and surface quality comparable to and even exceeding bulk diamond. Additionally, we have demonstrated that color centers within the membranes have sufficient optical and spin coherence for a broad range of applications in QIS. These diamond membranes can serve as a quantum material platform for a wide range of current and future research directions. The sub-wavelength thickness of the diamond membranes expands capabilities for nanophotonic integration. ${ }^{13}$ Additionally, this platform eases the fabrication of strain engineering ${ }^{56}$ and Stark tuning, ${ }^{57}$ which also provides an opportunity to study behavior of color centers under extreme environments such as strong strain or electrical fields. Moreover, the versatility afforded by the arbitrary carrier wafer

platform is vital for integrating diamond-based quantum systems to phononic, ${ }^{12,58}$ superconducting, and magnonic ${ }^{59}$ systems for quantum transduction in a hybrid geometry. ${ }^{60}$ Furthermore, $\mathrm{NV}^{-}$-based quantum sensing will benefit from the ease of integration with a number of interfaces, ranging from type-II superconductors to living cells. ${ }^{15,16}$ The isotopically purified, highly crystalline diamond membranes are also prime candidates for heat dissipation device studies ${ }^{61}$ and high quality engineered nanodiamonds synthesis. ${ }^{62,63}$

\section{Acknowledgement}

This work was primarily supported by the U.S. Department of Energy, Office of Science, Basic Energy Sciences, Materials Sciences and Engineering Division with support from the U.S. Department of Energy, Office of Science, National Quantum Information Science Research Centers. A. H. was partially supported by the University of Chicago Materials Research Science and Engineering Center, which is funded by the National Science Foundation under award number DMR2011854. This work made use of the Pritzker Nanofabrication Facility part of the Pritzker School of Molecular Engineering at the University of Chicago, which receives support from Soft and Hybrid Nanotechnology Experimental (SHyNE) Resource (NSF ECCS-2025633), a node of 
the National Science Foundation's National Nanotechnology Coordinated Infrastructure. This work also made use of the shared facilities at the University of Chicago Materials Research Science and Engineering Center, supported by the National Science Foundation under award number DMR-2011854. Funding was provided by the Boeing company and the University of Chicago Joint Task Force Initiative. J. K. and A. B. acknowledge support from the NSF Graduate Research Fellowship under grant no. DGE-1746045. The authors thank A. S. Greenspon for experimental help.

\section{References}

(1) Ohno, K.; Joseph Heremans, F.; Bassett, L. C.; Myers, B. A.; Toyli, D. M.; Bleszynski Jayich, A. C.; Palmstrøm, C. J.; Awschalom, D. D. Engineering shallow spins in diamond with nitrogen delta-doping. Applied Physics Letters 2012, 101, 082413.

(2) Sukachev, D. D.; Sipahigil, A.; Nguyen, C. T.; Bhaskar, M. K.; Evans, R. E.; Jelezko, F.; Lukin, M. D. Silicon-Vacancy Spin Qubit in Diamond: A Quantum Memory Exceeding 10 ms with Single-Shot State Readout. Phys. Rev. Lett. 2017, 119, 223602.

(3) Togan, E.; Chu, Y.; Trifonov, A. S.; Jiang, L.; Maze, J.; Childress, L.; Dutt, M. V. G.; Sørensen, A. S.; Hemmer, P. R.; Zibrov, A. S.; Lukin, M. D. Quantum entanglement between an optical photon and a solid-state spin qubit. Nature 2010, 466, 730-734.

(4) Rogers, L. J.; Jahnke, K. D.; Metsch, M. H.; Sipahigil, A.; Binder, J. M.; Teraji, T.; Sumiya, H.; Isoya, J.; Lukin, M. D.; Hemmer, P.; Jelezko, F. All-Optical Initialization, Readout, and Coherent Preparation of Single Silicon-Vacancy Spins in Diamond. Phys. Rev. Lett. 2014, $113,263602$.

(5) Robledo, L.; Childress, L.; Bernien, H.; Hensen, B.; Alkemade, P. F. A.; Hanson, R. Highfidelity projective read-out of a solid-state spin quantum register. Nature 2011, 477, $574-578$. 
(6) Metsch, M. H.; Senkalla, K.; Tratzmiller, B.; Scheuer, J.; Kern, M.; Achard, J.; Tallaire, A.; Plenio, M. B.; Siyushev, P.; Jelezko, F. Initialization and Readout of Nuclear Spins via a Negatively Charged Silicon-Vacancy Center in Diamond. Physical Review Letters 2019, 122, 190503.

(7) Sushkov, A.; Lovchinsky, I.; Chisholm, N.; Walsworth, R.; Park, H.; Lukin, M. Magnetic Resonance Detection of Individual Proton Spins Using Quantum Reporters. Physical Review Letters 2014, 113, 197601.

(8) Humphreys, P. C.; Kalb, N.; Morits, J. P. J.; Schouten, R. N.; Vermeulen, R. F. L.; Twitchen, D. J.; Markham, M.; Hanson, R. Deterministic delivery of remote entanglement on a quantum network. Nature 2018, 558, 268-273.

(9) Pompili, M.; Hermans, S. L. N.; Baier, S.; Beukers, H. K. C.; Humphreys, P. C.;

Schouten, R. N.; Vermeulen, R. F. L.; Tiggelman, M. J.; dos Santos Martins, L.; Dirkse, B.; Wehner, S.; Hanson, R. Realization of a multinode quantum network of remote solid-state qubits. Science 2021, 372, 259-264.

(10) DeVience, S. J.; Pham, L. M.; Lovchinsky, I.; Sushkov, A. O.; Bar-Gill, N.; Belthangady, C.; Casola, F.; Corbett, M.; Zhang, H.; Lukin, M.; Park, H.; Yacoby, A.; Walsworth, R. L. Nanoscale NMR spectroscopy and imaging of multiple nuclear species. Nature Nanotechnology 2015, 10, 129-134.

(11) Bhaskar, M. K.; Riedinger, R.; Machielse, B.; Levonian, D. S.; Nguyen, C. T.; Knall, E. N.; Park, H.; Englund, D.; Loncar, M.; Sukachev, D. D.; Lukin, M. D. Experimental demonstra-` tion of memory-enhanced quantum communication. Nature 2020, 580, 60-64.

(12) Maity, S.; Shao, L.; Bogdanovic, S.; Meesala, S.; Sohn, Y.-I.; Sinclair, N.; Pingault, B.; Chalupnik, M.; Chia, C.; Zheng, L.; Lai, K.; Loncar, M. Coherent acoustic control of a single` silicon vacancy spin in diamond. Nature Communications 2020, 11, 193. 
(13) Butcher, A.; Guo, X.; Shreiner, R.; Delegan, N.; Hao, K.; Duda, P. J.; Awschalom, D. D.; Heremans, F. J.; High, A. A. High-Q Nanophotonic Resonators on Diamond Membranes using Templated Atomic Layer Deposition of TiO2. Nano Letters 2020, 20, 4603-4609.

(14) Vermeulen, N.; Sipe, J. E.; Helt, L. G.; Thienpont, H. Opportunities for wavelength conversion with on-chip diamond ring resonators. Laser and Photonics Reviews 2012, 6, 793-801.

(15) Yip, K. Y.; Ho, K. O.; Yu, K. Y.; Chen, Y.; Zhang, W.; Kasahara, S.; Mizukami, Y.; Shibauchi, T.; Matsuda, Y.; Goh, S. K.; Yang, S. Measuring magnetic field texture in correlated electron systems under extreme conditions. Science 2019, 366, 1355 - 1359.

(16) Le Sage, D.; Arai, K.; Glenn, D. R.; DeVience, S. J.; Pham, L. M.; Rahn-Lee, L.; Lukin, M. D.; Yacoby, A.; Komeili, A.; Walsworth, R. L. Optical magnetic imaging of living cells. Nature 2013, 496, 486-489.

(17) Schreck, M.; Gsell, S.; Brescia, R.; Fischer, M. Ion bombardment induced buried lateral growth: the key mechanism for the synthesis of single crystal diamond wafers. Scientific Reports 2017, 7, 44462.

(18) Gallheber, B.-C.; Fischer, M.; Mayr, M.; Straub, J.; Schreck, M. Growth, stress, and defects of heteroepitaxial diamond on Ir/YSZ/Si(111). Journal of Applied Physics 2018, 123, 225302.

(19) Burek, M. J.; de Leon, N. P.; Shields, B. J.; Hausmann, B. J. M.; Chu, Y.; Quan, Q.; Zibrov, A. S.; Park, H.; Lukin, M. D.; Loncar, M. Free-Standing Mechanical and Photonic` Nanostructures in Single-Crystal Diamond. Nano Letters 2012, 12, 6084-6089.

(20) Høy Jensen, R.; Janitz, E.; Fontana, Y.; He, Y.; Gobron, O.; Radko, I. P.; Bhaskar, M.; Evans, R.; Rodríguez Rosenblueth, C. D.; Childress, L.; Huck, A.; Lund Andersen, U. CavityEnhanced Photon Emission from a Single Germanium-Vacancy Center in a Diamond Membrane. Physical Review Applied 2020, 13, 64016. 
(21) Wan, N. H.; Lu, T.-J.; Chen, K. C.; Walsh, M. P.; Trusheim, M. E.; De Santis, L.; Bersin, E. A.; Harris, I. B.; Mouradian, S. L.; Christen, I. R.; Bielejec, E. S.; Englund, D. Large-scale integration of artificial atoms in hybrid photonic circuits. Nature 2020, 583, 226-231.

(22) Ruf, M.; IJspeert, M.; van Dam, S.; de Jong, N.; van den Berg, H.; Evers, G.; Hanson, R. Optically Coherent Nitrogen-Vacancy Centers in Micrometer-Thin Etched Diamond Membranes. Nano Letters 2019, 19, 3987-3992.

(23) Aharonovich, I.; Lee, J. C.; Magyar, A. P.; Buckley, B. B.; Yale, C. G.; Awschalom, D. D.; $\mathrm{Hu}$, E. L. Homoepitaxial Growth of Single Crystal Diamond Membranes for Quantum Information Processing. Advanced Materials 2012, 24, OP54-OP59.

(24) Fuchs, P.; Jung, T.; Kieschnick, M.; Meijer, J.; Becher, C. A cavity-based optical antenna for color centers in diamond. arXiv e-prints 2021, arXiv:2105.10249.

(25) Gaathon, O.; Hodges, J. S.; Chen, E. H.; Li, L.; Bakhru, S.; Bakhru, H.; Englund, D.; Osgood, R. M. Planar fabrication of arrays of ion-exfoliated single-crystal-diamond membranes with nitrogen-vacancy color centers. Optical Materials 2013, 35, 361-365.

(26) Bray, K.; Regan, B.; Trycz, A.; Previdi, R.; Seniutinas, G.; Ganesan, K.; Kianinia, M.; Kim, S.; Aharonovich, I. Single Crystal Diamond Membranes and Photonic Resonators Containing Germanium Vacancy Color Centers. ACS Photonics 2018, 5, 4817-4822.

(27) Magyar, A. P.; Lee, J. C.; Limarga, A. M.; Aharonovich, I.; Rol, F.; Clarke, D. R.; Huang, M.;

$\mathrm{Hu}$, E. L. Fabrication of thin, luminescent, single-crystal diamond membranes. Applied Physics Letters 2011, 99, 81913.

(28) Piracha, A. H.; Rath, P.; Ganesan, K.; Kühn, S.; Pernice, W. H. P.; Prawer, S. Scalable Fabrication of Integrated Nanophotonic Circuits on Arrays of Thin Single Crystal Diamond Membrane Windows. Nano Letters 2016, 16, 3341-3347. 
(29) Vogelgesang, R.; Ramdas, A. K.; Rodriguez, S.; Grimsditch, M.; Anthony, T. R. Brillouin and Raman scattering in natural and isotopically controlled diamond. Physical Review B 1996, 54, 3989-3999.

(30) Lee, J. C.; Magyar, A. P.; Bracher, D. O.; Aharonovich, I.; Hu, E. L. Fabrication of thin diamond membranes for photonic applications. Diamond and Related Materials 2013, 33, 45-48.

(31) Ziegler, J. F.; Ziegler, M. D.; Biersack, J. P. SRIM - The stopping and range of ions in matter (2010). Nuclear Instruments and Methods in Physics Research Section B: Beam Interactions with Materials and Atoms 2010, 268, 1818-1823.

(32) Supporting Information.

(33) Sangtawesin, S. et al. Origins of Diamond Surface Noise Probed by Correlating Single-Spin Measurements with Surface Spectroscopy. Physical Review X 2019, 9, 31052.

(34) Naydenov, B.; Reinhard, F.; Lämmle, A.; Richter, V.; Kalish, R.; D’Haenens-Johansson, U. F. S.; Newton, M.; Jelezko, F.; Wrachtrup, J. Increasing the coherence time of single electron spins in diamond by high temperature annealing. Applied Physics Letters 2010, 97, 242511.

(35) Meynell, S. A.; McLellan, C. A.; Hughes, L. B.; Wang, W.; Mates, T. E.; Mukherjee, K.; Bleszynski Jayich, A. C. Engineering quantum-coherent defects: The role of substrate miscut in chemical vapor deposition diamond growth. Applied Physics Letters 2020, 117, 194001.

(36) Rose, B. C.; Huang, D.; Zhang, Z.-H.; Stevenson, P.; Tyryshkin, A. M.; Sangtawesin, S.; Srinivasan, S.; Loudin, L.; Markham, M. L.; Edmonds, A. M.; Twitchen, D. J.; Lyon, S. A.; de Leon, N. P. Observation of an environmentally insensitive solid-state spin defect in diamond. Science 2018, 361, 60 LP - 63. 
(37) Zomer, P. J.; Guimarães, M. H. D.; Brant, J. C.; Tombros, N.; van Wees, B. J. Fast pick up technique for high quality heterostructures of bilayer graphene and hexagonal boron nitride. Applied Physics Letters 2014, 105, 13101.

(38) Siew, Y. K.; Sarkar, G.; Hu, X.; Hui, J.; See, A.; Chua, C. T. Thermal Curing of Hydrogen Silsesquioxane. Journal of The Electrochemical Society 2000, 147, 335.

(39) Jung, T.; Kreiner, L.; Pauly, C.; Mücklich, F.; Edmonds, A. M.; Markham, M.; Becher, C. Reproducible fabrication and characterization of diamond membranes for photonic crystal cavities. physica status solidi (a) 2016, 213, 3254-3264.

(40) Ohno, K. Nanometer-scale engineering of shallow spins in diamond. Ph.D. thesis, University of California, Santa Barbara, 2014.

(41) Lee, C. L.; Gu, E.; Dawson, M. D.; Friel, I.; Scarsbrook, G. A. Etching and micro-optics fabrication in diamond using chlorine-based inductively-coupled plasma. Diamond and Related Materials 2008, 17, 1292-1296.

(42) Bhaskar, M.; Sukachev, D.; Sipahigil, A.; Evans, R.; Burek, M.; Nguyen, C.; Rogers, L.; Siyushev, P.; Metsch, M.; Park, H.; Jelezko, F.; Loncar, M.; Lukin, M. Quantum Nonlin-` ear Optics with a Germanium-Vacancy Color Center in a Nanoscale Diamond Waveguide. Physical Review Letters 2017, 118, 223603.

(43) Maity, S.; Shao, L.; Sohn, Y.-I.; Meesala, S.; Machielse, B.; Bielejec, E.; Markham, M.; Loncar, M. Spectral Alignment of Single-Photon Emitters in Diamond using Strain Gradient. Physical Review Applied 2018, 10, 24050.

(44) Iwasaki, T. et al. Germanium-Vacancy Single Color Centers in Diamond. Scientific Reports $2015,5,12882$. 
(45) Nguyen, C. T.; Sukachev, D. D.; Bhaskar, M. K.; Machielse, B.; Levonian, D. S.; Knall, E. N.; Stroganov, P.; Chia, C.; Burek, M. J.; Riedinger, R.; Park, H.; Loncar, M.; Lukin, M. D. An integrated nanophotonic quantum register based on silicon-vacancy spins in diamond. Physical Review B 2019, 100, 165428.

(46) Siyushev, P. et al. Optical and microwave control of germanium-vacancy center spins in diamond. Physical Review B 2017, 96, 81201.

(47) Rugar, A. E.; Dory, C.; Sun, S.; Vuckovi` c, J. Characterization of optical and spin properties` of single tin-vacancy centers in diamond nanopillars. Physical Review B 2019, 99, 205417.

(48) Barry, J. F.; Schloss, J. M.; Bauch, E.; Turner, M. J.; Hart, C. A.; Pham, L. M.; Walsworth, R. L. Sensitivity optimization for NV-diamond magnetometry. Reviews of Modern Physics 2020, 92, 15004.

(49) Myers, B.; Das, A.; Dartiailh, M.; Ohno, K.; Awschalom, D.; Bleszynski Jayich, A. Probing Surface Noise with Depth-Calibrated Spins in Diamond. Physical Review Letters 2014, 113, 27602.

(50) Yamamoto, T. et al. Extending spin coherence times of diamond qubits by high-temperature annealing. Physical Review B 2013, 88, 75206.

(51) Wang, Z.-H.; Takahashi, S. Spin decoherence and electron spin bath noise of a nitrogenvacancy center in diamond. Physical Review B 2013, 87, 115122.

(52) Fukami, M.; Candido, D. R.; Awschalom, D. D.; Flatté, M. E. Opportunities for longrange magnon-mediated entanglement of spin qubits via on- and off-resonant coupling. 2021, $\operatorname{arXiv:2101.09220.~}$ 
(53) Candido, D. R.; Fuchs, G. D.; Johnston-Halperin, E.; Flatté, M. E. Predicted strong coupling of solid-state spins via a single magnon mode. Materials for Quantum Technology 2020, 1, 011001.

(54) Mittiga, T.; Hsieh, S.; Zu, C.; Kobrin, B.; Machado, F.; Bhattacharyya, P.; Rui, N. Z.; Jarmola, A.; Choi, S.; Budker, D.; Yao, N. Y. Imaging the Local Charge Environment of NitrogenVacancy Centers in Diamond. Phys. Rev. Lett. 2018, 121, 246402.

(55) Ovartchaiyapong, P.; Lee, K. W.; Myers, B. A.; Jayich, A. C. B. Dynamic strain-mediated coupling of a single diamond spin to a mechanical resonator. Nature Communications 2014, 5,4429 .

(56) Meesala, S. et al. Strain engineering of the silicon-vacancy center in diamond. Physical Review B 2018, 97, 205444.

(57) Aghaeimeibodi, S.; Riedel, D.; Rugar, A. E.; Dory, C.; Vuckovic, J. Electrical tuning of tinvacancy centers in diamond. arXiv e-prints 2021, arXiv:2105.10249.

(58) Chen, H.; Opondo, N. F.; Jiang, B.; MacQuarrie, E. R.; Daveau, R. S.; Bhave, S. A.; Fuchs, G. D. Engineering Electron-Phonon Coupling of Quantum Defects to a Semiconfocal Acoustic Resonator. Nano Letters 2019, 19, 7021-7027.

(59) Awschalom, D. D. et al. Quantum Engineering With Hybrid Magnonics Systems and Materials. IEEE Transactions on Quantum Engineering 2021, 1.

(60) Kurizki, G.; Bertet, P.; Kubo, Y.; Mølmer, K.; Petrosyan, D.; Rabl, P.; Schmiedmayer, J. Quantum technologies with hybrid systems. Proceedings of the National Academy of Sciences 2015, 112, 3866 LP - 3873.

(61) Wei, L.; Kuo, P. K.; Thomas, R. L.; Anthony, T. R.; Banholzer, W. F. Thermal conductivity of isotopically modified single crystal diamond. Physical Review Letters 1993, 70, 37643767. 
(62) Andrich, P.; Alemán, B. J.; Lee, J. C.; Ohno, K.; de las Casas, C. F.; Heremans, F. J.; Hu, E. L.; Awschalom, D. D. Engineered Micro- and Nanoscale Diamonds as Mobile Probes for HighResolution Sensing in Fluid. Nano Letters 2014, 14, 4959-4964.

(63) Hruszkewycz, S. O.; Cha, W.; Andrich, P.; Anderson, C. P.; Ulvestad, A.; Harder, R.; Fuoss, P. H.; Awschalom, D. D.; Heremans, F. J. In situ study of annealing-induced strain relaxation in diamond nanoparticles using Bragg coherent diffraction imaging. APL Materials 2017, 5, 26105. 


\title{
Supporting Information: Tunable and Transferable Diamond Membranes for Integrated Quantum Technologies
}

\author{
Xinghan Guo ${ }^{1}$, Nazar Delegan ${ }^{1,2}$, Jonathan C. Karsch ${ }^{1}$, Zixi Li ${ }^{1}$, \\ Tianle Liu ${ }^{3}$, Robert Shreiner ${ }^{3}$, Amy Butcher ${ }^{1}$, David D. Awschalom ${ }^{1,2,3}$, \\ F. Joseph Heremans ${ }^{1,2}$, Alexander A. High ${ }^{1,2 *}$ \\ ${ }^{1}$ Pritzker School of Molecular Engineering, University of Chicago, Chicago, IL 60637, USA \\ ${ }^{2}$ Center for Molecular Engineering and Materials Science Division, \\ Argonne National Laboratory, Lemont, IL 60439, USA \\ ${ }^{3}$ Department of Physics, University of Chicago, Chicago, IL 60637, USA \\ *E-mail: ahigh@uchicago.edu
}

\section{Diamond membrane fabrication procedure}

\subsection{Membrane synthesis via the graphitized underlayer formation}

Standard $3 \mathrm{~mm}$ by $3 \mathrm{~mm}$ by $0.25 \mathrm{~mm}$ single crystal, optical grade diamond substrates (Element $\mathrm{Six}, \leq 1 \mathrm{ppm}[\mathrm{N}]$ ) are used for the membrane synthesis. These are initially fine-polished to a surface $\mathrm{Rq}$ of $\leq 0.3 \mathrm{~nm}$ (Syntek LLC.) as to minimize morphological inconsistencies (see Figure $\mathrm{S} 1$ (a)). Next, the samples are implanted with ${ }^{4} \mathrm{He}^{+}$ions (CuttingEdge Ions LLC.) at $150 \mathrm{keV}$ to create a graphitized layer at $\approx 410 \mathrm{~nm}$ depth. This is done at an incidence angle of $7^{\circ}$ to avoid ion channeling. The dose is set to $5 \times 10^{16} \mathrm{~cm}^{-2}$, which is closely above the graphitization threshold as to minimize crystal damage (see section 1.5). In this work, a threestep annealing process was employed after the implantation: a $400{ }^{\circ} \mathrm{C}$ soak for $8 \mathrm{~h}$, followed by a $800{ }^{\circ} \mathrm{C}$ soak for $8 \mathrm{~h}$, completed with a $1200{ }^{\circ} \mathrm{C}$ anneal for $2 \mathrm{~h} .{ }^{1}$ This was done in a forming gas environment (Ar: $\mathrm{H}_{2}$ of $96: 4$ ). The implantation and annealing had no negative impact on the surface roughness (see Figure S1 (b)). The phase transition of the carbon bonds during membrane formation is studied via room temperature Raman spectroscopy (see section 2.2).

\subsection{PE-CVD diamond overgrowth and $\delta$-doping of ${ }^{15} \mathrm{~N}$}

Homoepitaxial PE-CVD of diamond is performed in a custom configured SEKI DIAMOND SDS6350 system. Before loading into the growth chamber, the diamond substrates are chemically tri-acid cleaned (see section 1.7). The diamond sample is placed on a grooved 
molybdenum substrate to keep it flat with the rest of the plasma exposed surface, allowing for a uniform growth rate across the sample, while minimizing edge effects. The chamber is pumped down to a $2 \times 10^{-8}$ Torr to $5 \times 10^{-8}$ Torr base pressure in order to reduce background contamination. Thereafter, high purity $\mathrm{H}_{2}(99.999999 \%$ chemical purity) is introduced into the chamber, with the gas flow ratios constant and pumping rate monitored as to maintain a constant process pressure of 25 Torr. Throughout the process the microwave power is maintained at 900 $\mathrm{W}\left(11.5 \mathrm{Wmm}^{-2}\right)$. Before introducing the carbon precursor, the substrate is heated to a $500{ }^{\circ} \mathrm{C}$ or $700{ }^{\circ} \mathrm{C}$ setpoint which translates to a pyrometer assessed plasma temperature of $540(10){ }^{\circ} \mathrm{C}$ or $740(10){ }^{\circ} \mathrm{C}$, respectively. The $\mathrm{H}_{2}$-only plasma conditions are maintained for $20 \mathrm{~min}\left(500{ }^{\circ} \mathrm{C}\right)$ and $1 \mathrm{~h}\left(700{ }^{\circ} \mathrm{C}\right)$ as to etch away any residual surface carbonaceous contaminants - the approximated $\mathrm{sp}^{3}$ etch depth is $\leq 1 \mathrm{~nm} .{ }^{2}$ Thereafter, ${ }^{12} \mathrm{CH}_{4}(99.99999 \%$ chemical purity, 99.99 at.\% isotopic purity) is introduced as the carbon precursor, this injection is substantiated in-situ via increase of the pyrometer detected substrate surface temperature to $765(10){ }^{\circ} \mathrm{C}\left(580(10){ }^{\circ} \mathrm{C}\right.$ for the $500{ }^{\circ} \mathrm{C}$ set-point grown substrate). The methane-to-hydrogen ratio is maintained constant at $0.05 \%\left(\mathrm{H}_{2}:{ }^{12} \mathrm{CH}_{4}=400 \mathrm{sccm}: 0.2 \mathrm{sccm}\right)$ as to ensure step-flow growth. ${ }^{3,4}$ Growth rates for the $700{ }^{\circ} \mathrm{C}$ and $500{ }^{\circ} \mathrm{C}$ membranes were determined to be 6.2(4) $\mathrm{nmh}^{-1}$ and 9.3(8) $\mathrm{nmh}^{-1}$, respectively (see section 2.3). The reduction in growth rate is explained by an increased surface mobility and desorption of precursor adatoms ${ }^{5}$ and likely due to a non-linear reduction of nucleation sites.

In-situ nitrogen doping is accomplished by introducing $0.06 \mathrm{sccm}$ of ${ }^{15} \mathrm{~N}_{2}$ gas $(99.99 \%$ chemical purity, 99.9 at.\% isotopic purity) for 2 min during the growth phase. This creates a $36 \mathrm{~nm}$ depth localized layer with a SIMS quantified $\left[{ }^{15} \mathrm{NV}^{-}\right]$density of 30.8(57) ppb (see section 4.5). Thereafter, a cap layer is overgrown to encapsulate the $\delta$-doped layer as deep in the membrane as desired. Subsequent irradiation and annealing allows the conversion of this layer into $\approx 0.9 \mathrm{ppb}$ of $\left[{ }^{15} \mathrm{NV}^{-}\right]$, resulting in an average color center creation efficiency of $\leq 2.5 \%$, in agreement with previous studies (see section 4.5 ). ${ }^{3}$

\subsection{Membrane patterning and EC etching}

Atomic layer deposition (ALD) is used to deposit $25 \mathrm{~nm}$ of $\mathrm{Al}_{2} \mathrm{O}_{3}$ on the overgrown membrane samples. This oxide layer serves as selective hard mask for subsequent ICP etching. Thereafter, a photoresist layer (AZ MiR 703) is applied to the sample, followed by a lithography exposure (Heidelberg MLA150, $375 \mathrm{~nm}$ laser, with a $150 \mathrm{mJcm}^{-2}$ dose). A total of 56 square-shaped membranes are patterned on a single substrate. Next, the sample is developed (AZ 300 MIF) and two-step etched in a chlorine-based ICP chamber (Plasma - Therm, $\mathrm{Al}_{2} \mathrm{O}_{3}$ etched with $\mathrm{BCl}_{3}$ chemistry first, with diamond subsequently etched in $\mathrm{O}_{2}$ chemistry, see Table $\mathrm{S} 2$ ). This step exposes the underlying graphitic layer. After etching, the sample is ultrasonicated in $80{ }^{\circ} \mathrm{C}$ NMethyl-2-pyrrolidone (NMP) solution and placed in a buffered oxide etch (BOE) solution to remove excess resist and oxide layers. 
For the EC etching step, the sample is held by double side tapes at the bottom of a DI waterfilled petri dish which is mounted on a probe station (Signatone S1160). Unlike previous studies which required customized platinum tips, ${ }^{6}$ we use commercially available tungsten tips (Signatone SE-T) as the cathode and palladium tips (Signetone SE-P) as the anode (see Figure S3 (b)). These tips are mounted to 3-axis micropositioners (Signatone S926), which are connected to a DC power supply (B\&K Precision 1787B). Typically, a voltage between $15 \mathrm{~V}$ and $36 \mathrm{~V}$ is applied to undercut the membranes, with larger undercut areas requiring larger potentials and longer time. The EC etch is visually tracked, with the voltage cut at the moment that the desired undercut tether has been achieved. This process, while relatively simple, requires constant supervision as the final tether feature size is typically less than $50 \mu \mathrm{m}$ in any direction. Practically speaking, at the typical voltages quoted above, this translates to an etching time of $\approx 30$ min per membrane (see Figure $S 3$ (c)). This processing step is a great candidate for future automation.

\subsection{Membrane transfer and post-processing}

Prior to the membrane dry transfer, the intended carrier wafers or devices are prepared with predefined structures and features (see section 1.8). In this work, we use $7 \mathrm{~mm}$ by $7 \mathrm{~mm}$ fused silica $\left(\mathrm{SiO}_{2}, \mathrm{JGS} 2\right.$ grade) and thermal oxide $\left(280 \mathrm{~nm} \mathrm{SiO} 2 \_\mathrm{Si}\right)$ wafers. Alignment markers and $5 \mu \mathrm{m}$ deep trenches are patterned and etched into substrates to suspend part of the transferred membrane (see Figure S5 (a)). In this way, both surfaces of the membranes are exposed, enabling double-sided surface termination via wet-chemical or dry processes with acid cleaning and oxygen annealing used in this work. As for the dry-transfer itself, we start by mounting the diamond substrate on a transfer station rotational chuck (Signatone S1160). Parallel to this, we prepare a PDMS/PC stamp on a glass slide and mount it into a computer-aided micropositioner (Signatone CAP - 946). Next, we slowly bring the stamp down and allow it to fully adhere to the target membrane. With a quick movement of the now-adhered stamp, we break the membrane from the tether. Then, we replace the substrate on the chuck with a second PDMS stamp on a glass slide. The detached membrane can be transferred to this second stamp by slowly bringing the PDMS/PC stamp into contact with the "stickier" PDMS-only stamp and lifting the PDMS/PC stamp afterwards. Finally, with the membrane fully adhered to the PDMSonly stamp, we use the micropositioner to mount the membrane to an HSQ-covered carrier wafer on the chuck. After careful alignment, we gradually bring the stamp down until the membrane is fully attached to a freshly spin-coated HSQ layer (14\% HSQ in methyl isobutyl ketone (MIBK) solution, DisChem Inc.), and then slowly release the stamp. The membrane is left on the carrier wafer due to stronger adhesion from the HSQ polymer and the residual MIBK solvents (see Figure S4).

After membrane transfer, the carrying wafer is annealed at $600{ }^{\circ} \mathrm{C}$ for $8 \mathrm{~h}$ in Ar environment. The HSQ adhesion layer fully collapses during the annealing and forms a nearly strain-free $\approx 250 \mathrm{~nm}$ thick layer. This thickness can be tuned by using different concentration of HSQ 
solvents or by varying the spinning parameters. In the case of partially suspended membranes, we applied a vapor HF treatment (Memsstar ORBIS ALPHA) to fully remove the HSQ in the trench (see section 2.4). Additionally, in order to etch the original damaged underlayer (now on the top face of the membrane), improve surface morphology, and tune the final thickness, we submit the mounted membrane to a three-step ICP process (see Table S2). ${ }^{1}$ We start with the "Ar/ $\mathrm{Cl}_{2}$ " recipe ( 2 min to $5 \mathrm{~min}$, depending on the target thickness) to polish the surface, then we use the " $\mathrm{O}_{2} / \mathrm{Cl}_{2}$ " recipe (30 s to $90 \mathrm{~s}$ ) to remove most of the chlorine-based compounds on the diamond surface. Finally, we apply a $30 \mathrm{~s}$ " $\mathrm{O}_{2}$ " etch to remove the residual chlorine compounds and correctly terminate the surface. In order to avoid chemical contamination, etching steps are separated by multiple pump-purge cycles. AFM characterizations are added post-etching to ensure no micromasking occured during ICP processes. ${ }^{7}$ The membrane thickness is monitored via either a profilometer (Bruker DektakXT) or an ellipsometer (Horiba UVISEL2) (see section 2.3).

\subsection{Additional information on ion implantation}

Two distinct ion implantation runs were performed in this work: ${ }^{4} \mathrm{He}^{+}$ion implantation for the creation of the graphitized underlayer and ${ }^{14} \mathrm{~N}^{+},{ }^{28} \mathrm{Si}^{+},{ }^{74} \mathrm{Ge}^{+}$, and ${ }^{120} \mathrm{Sn}^{+}$ion implantation for the creation of color centers. Both implantation processes were performed at CuttingEdge Ions, LLC.

To achieve the formation of the graphitized underlayer, a low energy ${ }^{4} \mathrm{He}^{+}$-ion implantation into the diamond substrates was done as depicted in Figure 1 (a). This step forms a depthlocalized graphitized underlayer $\approx 410 \mathrm{~nm}$ deep via a damage-induced phase transition from $\mathrm{sp}^{3}$ to $\mathrm{sp}^{2}$. The implantation energy was kept at $150 \mathrm{keV}$ to minimize crystal damage along the ${ }^{4} \mathrm{He}^{+}$trajectory. The dose was set to $5 \times 10^{16} \mathrm{~cm}^{-2}$, which is above the graphitization threshold, but minimized to avoid unnecessary crystal damage. Separate implantation tests showed that lower doses of $\leq 2 \times 10^{16} \mathrm{~cm}^{-2}$ were insufficient to generate a reliable graphitized layer, with the diamond substrate remaining transparent. Therefore, the desired damage threshold was established to be between $2 \times 10^{16} \mathrm{~cm}^{-2}$ to $5 \times 10^{16} \mathrm{~cm}^{-2}$.

We use the Stopping and Range of Ions in Matter software (SRIM) ${ }^{8}$ to estimate the ion implantation depth and straggle. For the simulations, the density of carbon is set to $3.51 \mathrm{gcm}^{-3}$, and the incidence angle is $7^{\circ}$ to avoid ion channeling. For color center incorporation, we simultaneously implanted ${ }^{14} \mathrm{~N}^{+},{ }^{28} \mathrm{Si}^{+},{ }^{74} \mathrm{Ge}^{+}$and ${ }^{120} \mathrm{Sn}^{+}$at a dose of $2 \times 10^{8} \mathrm{~cm}^{-2}$ in order to achieve an individual-level color center density. Details about the implantation parameters and simulated depths and straggles are shown in Table $\mathrm{S} 1$.

All of the implantation steps were followed with the three-step $1200{ }^{\circ} \mathrm{C}$ anneal ${ }^{1}$ (see section 1.1) to form the graphitized layer and color centers, in the case of the $\mathrm{He}^{+}$and color center implantation, respectively. 


\subsection{Electron irradiation}

For the synthesis of delta-doped color centers, an electron radiation of $10 \times 10^{18} \mathrm{~cm}^{-2}$ at $2 \mathrm{MeV}$ at the low energy accelerator facility (LEAF) at Argonne National Laboratory was performed to form vacancies throughout the diamond crystal. This was followed by a $6 \mathrm{~h}$ vacancy mobilization anneal at $850^{\circ} \mathrm{C}$ in forming gas $\left(96: 4\right.$ of $\left.\mathrm{Ar}: \mathrm{H}_{2}\right)$.

\subsection{Surface cleaning and termination}

In order to remove contamination and passivate the surface, acid-related surface cleanings and terminations are applied multiple times during membrane synthesis. Firstly, diamond substrates receive a tri-acid cleaning $\left(1: 1: 1\right.$ nitric, sulfuric, and perchloric acid) at $200{ }^{\circ} \mathrm{C}$ for $2 \mathrm{~h}$ right before the overgrowth to eliminate surface contamination ${ }^{9}$. Secondly, the diamond membrane template receives a di-acid cleaning ( $1: 1$ nitric and sulfuric acid) at $225^{\circ} \mathrm{C}$ for $2 \mathrm{~h}$ after every transfer step to prevent accumulated contamination induced by EC etching or PDMS/PC stamping. Finally, before optical measurements, membrane samples receive another di-acid cleaning and an optional oxygen termination, depending on the type of color centers being characterized. For group IV centers, we only apply the di-acid clean procedure. For NV center measurements, an additional oxygen termination is applied following di-acid cleaning, which includes a $4 \mathrm{~h}$ annealing at $400{ }^{\circ} \mathrm{C}$ in $\mathrm{O}_{2}$ atmosphere, with 5 min rinse in freshly prepared Piranha solution ( $3: 1$ sulfuric acid and hydrogen peroxide) before and after the annealing. The oxygen termination is intended for optimizing the $\mathrm{NV}^{0}$ to $\mathrm{NV}^{-}$conversion and stability ${ }^{10}$. The incorporation of higher temperature oxygen annealing while avoiding surface etching is an ongoing study and beyond the scope of this work.

\subsection{Carrier wafer preparation}

In this work, we dice thermal oxide and fused silica wafers to $7 \mathrm{~mm}$ by $7 \mathrm{~mm}$ squares (Disco DAD3240) as carrier wafers. Some wafers receive $\approx 5 \mu \mathrm{m}$ trench fabrication on the surface to allow membranes to receive double-side surface treatment and obtain low background fluorescence during optical measurements.

In order to etch the trenches, we deposit a $\approx 6 \mu \mathrm{m}$ photoresist layer (AZ 4620) as the etching mask. The spinning speed is set to $500 \mathrm{rpm}$ for $10 \mathrm{~s}$ and then $4000 \mathrm{rpm}$ for $50 \mathrm{~s}$, with a ramping rate of $100 \mathrm{rpms}^{-1}$ and $2000 \mathrm{rpms}^{-1}$, respectively. After resist spinning and pre-baking, the sample is lithographically exposed (Heidelberg MLA150, $405 \mathrm{~nm}$ laser with $320 \mathrm{mJcm}^{-2}$ dosage), followed by development in AZ 400K 1:4 solution. We use fluorine-based ICP (Plasma - Therm) for $\mathrm{SiO}_{2}$ and $\mathrm{Si}$ etching. After etching, samples are ultrasonicated in $80^{\circ} \mathrm{C} \mathrm{N}$-Methyl2pyrrolidone (NMP) solution to remove the resist. 


\subsection{Additional discussions on diamond membrane fabrication}

The membrane creation process highlighted herein advances scalable diamond quantum research in several important ways. While the process requires initial diamond substrates with sufficient crystallinity and smoothness, it is agnostic to moderate impurity and defect concentrations within the substrate. This eliminates the need for highly purified bulk diamond substrates, which are costly and scarce. Additionally, while bulk diamond crystals are commercially grown and available for purchase in a limited range of dimensions, the size and the shape of the membranes are fully defined and can be tailored to specific applications, with the maximum size only limited by the substrate dimensions. While our choice of membrane size $(200 \mu \mathrm{m} \times 200 \mu \mathrm{m})$ is well motivated by scientific applications, such delaminations over millimeter size have been achieved ${ }^{11}$, and there are no technical barriers to realizing larger dimensions with our technique.

\section{Material characterization}

\subsection{AFM characterization}

We extensively apply AFM measurements (Bruker Dimension Icon) to monitor the surface morphology throughout the synthesis steps. The scanning ranges and resolutions in both directions are set to $10 \mu \mathrm{m}$ and 512 lines (except for Figure S1 (f), $5 \mu \mathrm{m}$ with 256 lines) to observe the surface morphology and growth- or etching-related features.

The surface maps of overgrown and back-etched diamond membranes are shown in Figure 2 (a-c) of the main text, with maps of other key steps shown in Figure S1. Since the as-purchased diamond substrates are only roughly polished $(R a \leq 30 \mathrm{~nm})$, we applied another fine-polish (Syntek LLC.) step to reduce the roughness to $R q=0.30 \mathrm{~nm}(R a=0.20 \mathrm{~nm})$, as shown in Figure $S 1$ (a). The final surface miscut, relative to the crystallographic (001) axis, is specified to $\leq 3^{\circ}$. Unfortunately, while this value is relatively consistent within a single substrate batch, it can vary between substrate lots, affecting the effective implantation angle and overgrowth characteristics ${ }^{4}$.

The $\mathrm{He}^{+}$-graphitized and subsequently annealed substrate is shown in Figure S1 (b), with the same level of surface morphology $(R q=0.27 \mathrm{~nm})$. Figure $\mathrm{S} 1$ (c) exhibits the $40 \mathrm{~h}$ overgrown diamond surface with a pre-growth 60 min hydrogen plasma etch. The inconsistency of surface roughness under the same duration of hydrogen plasma etch (see Figure 2 (b)) may come from the residual surface strain from fine polishing. A surface strain release step ${ }^{1}$ after the fine-polish should improve this in future iterations of the fabrication process. Another $40 \mathrm{~h}$ growth without proper surface preparation is shown in Figure S1 (d). Residual contamination on the diamond surface generates growth defects (such as pits or pyramids) along the process. Figure S1 (e) shows the front side of the diamond membrane after the dry transfer. The excess roughness $(R q$ $=1.17 \mathrm{~nm}$ ) comes from the straggle of the $\mathrm{He}^{+}$implantation and can be reduced by subsequent " $\mathrm{Ar} / \mathrm{Cl}_{2}$ " etching. It is crucial to apply " $\mathrm{Ar} / \mathrm{Cl}_{2}$ " to prevent roughness deterioration. Figure S1 
(f) shows the front side of the membrane if the $\mathrm{O}_{2}$ etching is applied directly, where the surface deteriorates noticeably due to the preferential etching nature of an $\mathrm{O}_{2}$-based plasma on crystallographic defects and polish-induced damage.

\subsection{Raman characterization}

Throughout the process, we applied Raman spectroscopy (Horiba Scientific LabRAM HR Evolution) to investigate both the phase of the carbon during membrane formation and the diamond crystal quality. A $633 \mathrm{~nm}$ excitation laser is used instead of the more popular $532 \mathrm{~nm}$ to avoid broad fluorescence from $\mathrm{NV}^{-}$centers in the substrates. We use the finest grating (1800 $\mathrm{grmm}^{-1}$ ) for data collection, with a quoted resolution of $\approx 0.3 \mathrm{~cm}^{-1}$. The resulting peak position uncertainty is caused by this instrument resolution, while the linewidth uncertainty comes from the fittings. The pinhole of the Raman confocal microscope is set to either $30 \%$ or $50 \%$ to reduce artificial broadening.

To effectively evaluate the crystal quality of our overgrown membranes, we used a reference diamond for the Raman measurements. In order to achieve the cleanest Raman signature, we chose an electronic grade single crystal diamond from Element Six. Furthermore, we ICPetched the top $\approx 5 \mu \mathrm{m}$ of the reference sample, and subsequently annealed the substrate in order to remove the residual strain induced by surface polish. The etching procedure is described in ${ }^{12}$, with our exact process flow detailed in Table $\mathrm{S} 2$.

Figure S2 (a) shows the comparison of implanted diamond substrates pre- and post-annealing. Prior to annealing, only a weak diamond peak from the back substrate and low $\mathrm{sp}^{3}$ amorphous carbon features are observed at $\approx 1500 \mathrm{~cm}^{-1} 13$. Post annealing, three peaks are observed. The $\approx 1595 \mathrm{~cm}^{-1}$ and $\approx 2268 \mathrm{~cm}^{-1}$ peaks are strongly correlated with high $\mathrm{sp}^{3}$ amorphous carbon composite ${ }^{13}$, while the $\approx 1325 \mathrm{~cm}^{-1}$ peak represents the partially recovered top membrane layer ${ }^{14}$.

Figure S2 (b) shows the Raman peak of a $110 \mathrm{~nm}$ thick, $\approx 250 \mathrm{~nm}$ overgrowth diamond membrane grown in high temperature condition for $40 \mathrm{~h}$. The sample is named as " $\mathrm{N}$ doped membrane" because of the in-situ doping of ${ }^{15} \mathrm{~N}$ during overgrowth. The Raman linewidth of this membrane $\left(1.486(14) \mathrm{cm}^{-1}\right)$ is slightly broader than the low temperature one. It is likely that this slight broadening of $0.11 \mathrm{~cm}^{-1}$ originates from the higher heterogeneity of the crystal quality due to slight change in growth conditions from the delta doping and higher synthesis temperatures.

\subsection{Membrane thickness and growth rate characterization}

In this work, we applied two different approaches to ascertain the thicknesses of the membranes. For the growth rate analysis and thickness-insensitive applications, we used a profilometer (Bruker DektakXT) to measure the height difference between the diamond membranes and the carrier wafers supporting them. For thickness-sensitive applications, we use an ellipsometer (Horiba UVISEL2) to optically monitor the thickness during etching processes. 
For the profilometry characterizations the height limit is set to $6.5 \mu \mathrm{m}$ and the typical scanning length is set to $500 \mu \mathrm{m}$ with scanning time $\approx 200 \mathrm{~s}$. The scanning trajectory covers the full length of the membrane for a more accurate value. This measurement is launched after the HSQ annealing and before the "Ar$/ \mathrm{Cl}_{2}$ " etching. According to the characterization results, transferred membranes without overgrowth are 340(5) nm thick. The thicknesses of the $40 \mathrm{~h}$ overgrown membranes are $680 \mathrm{~nm}$ to $740 \mathrm{~nm}$ (low growth temperature of $500{ }^{\circ} \mathrm{C}$ ) and $570 \mathrm{~nm}$ to $600 \mathrm{~nm}\left(700{ }^{\circ} \mathrm{C}\right.$ growth temperature), resulting in growth rates of 9.3(8) $\mathrm{nmh}^{-1}$ and 6.2(4) $\mathrm{nmh}^{-1}$, respectively (growth rate uncertainty from multiple membranes measured). For thickness-insensitive applications, the ICP etching recipe for each membrane is calculated from measured initial thickness, the target thickness, and the ICP etching rates (Table S2).

We also used profilometry characterization to ascertain the overall membrane height and flatness. A scan across a ${ }^{15} \mathrm{~N}$-doped diamond membrane after the transfer is shown in Figure S5 (e). The result indicates an average membrane thickness of 603(10) nm before the back etching. This value shouldn't be affected by the back etching process as the ICP etching rate is uniform within the area of the membrane. Due to the angle between the membrane edge and the scanning direction, the length of the membrane in this figure seems to be longer than $200 \mu \mathrm{m}$. Since the scanning result includes some local height variation on the membrane, the real flatness should be less than $10 \mathrm{~nm}$, which indicates a very uniform thickness across the whole membrane area.

This excellent uniformity is crucial for future device fabrication and potential applications.

For thickness-sensitive applications, we used the ellipsometry scanning to the diamond membranes. This technique was firstly applied in nanophotonic integration studies ${ }^{15}$. The scanning area is set to $85 \mu \mathrm{m} \times 35 \mu \mathrm{m}$ and is carefully aligned to the membrane surface. The scanning step is set to $\approx 0.05 \mathrm{eV}$ with long probing time at each step $(\approx 1 \mathrm{~s})$ to reduce the fitting error. To fit the diamond layer, we use Cauchy's equation for transparent material

$$
n(\lambda)=A+\frac{B \cdot 10^{4}}{\lambda^{2}}+\frac{C \cdot 10^{9}}{\lambda^{4}},
$$

where $A=2.378, B=1.300$ and $C=0.000$, extracted from ${ }^{16}$. By using this model, we are able to get a $\leq 4 \mathrm{~nm}$ height uncertainty for $\leq 50 \mathrm{~nm}$ membranes.

\subsection{HSQ fluorescence analysis and vapor HF treatment}

As the adhesion layer between diamond membranes and carrying wafers, the HSQ introduces extra fluorescence under laser excitation. These additional counts mostly span from $560 \mathrm{~nm}$ to $850 \mathrm{~nm}$ and can affect the PL measurements to some extent, depending on the types of color centers being measured.

The resonant PLE measurements are not affected due to their narrow optical linewidths, which allows for the use of exceptionally low excitation power $(\leq 100 \mathrm{nW})$ and realize a large contrast of $\approx 30$ against the background and APD dark counts. Therefore, for experiments and technologies that rely on resonant interactions, such as those proposed for quantum networking, 
the HSQ background will have minimal impact on device performance. The off-resonant photoluminescence measurements on group IV color centers (such as $\mathrm{GeV}^{-}$) are mainly used for identifying the center locations, and are not critical for their performance in quantum networking. Due to the high Debye-Waller factors, group IV centers are typically measured with a narrow bandpass filter, which filters out most of the fluorescence from HSQ. With 10 $\mathrm{mW}$ excitation $(532 \mathrm{~nm})$ and appropriate filter sets, the HSQ fluorescence is $\approx 10 \mathrm{kHz}$ to $20 \mathrm{kHz}$, which is less than the ZPL counts from individual centers $(\approx 20 \mathrm{kHz}$ to $40 \mathrm{kHz}$, shown in Figure 3 (b) of the main text). Therefore, the presence of HSQ does not fundamentally hinder the optical characterization of $\mathrm{GeV}^{-}$centers or preclude the usage of $\mathrm{GeV}^{-}$in quantum networking applications, the primary technology driver for group IV color centers. Despite its limited impact, the HSQ background can be further reduced as wish via membrane suspension $(\approx 2 \mathrm{kHz}$ to $5 \mathrm{kHz})$ or vapor $\mathrm{HF}$ undercut $(\approx 0.5 \mathrm{kHz}$ to $1.5 \mathrm{kHz})$.

For $\mathrm{NV}^{-}$centers, the usage of a single longpass filter $(561 \mathrm{~nm})$ leads to a relatively high HSQ counts ( $300 \mathrm{kHz}$ to $500 \mathrm{kHz}$ under $10 \mathrm{~mW}$ green laser excitation), which hinders the PL and ODMR measurements. Therefore, $\mathrm{NV}^{-}$measurements benefit from reducing the HSQ background counts. In this work, we combined the suspended membrane fabrication (discussed in section 1.8) and the vapor HF treatment (discussed below) to reduce the total background to $15 \mathrm{kHz}$ to $30 \mathrm{kHz}$, which is comparable to the bulk diamond background. Other potential methods to reduce the HSQ background, such as HSQ patterning before transfer, usage of triacid clean to oxidize the HSQ layer, or material substitution with less optical fluorescence, will be explored in future studies.

The vapor HF treatment was used optionally to further minimize the background fluorescence from the HSQ underneath the suspended membrane region. During the vapor HF treatment, the chamber pressure is set to 24.5 Torr, with $\mathrm{H}_{2} \mathrm{O}$ flow at $18.9 \mathrm{mLmin}^{-1}$ and $\mathrm{HF}$ flow at $40 \mathrm{sccm}$. Figure S5 (a) shows a bare carrier wafer after trench fabrication, and Figure S5 (b) shows one of the trenches after the HSQ deposition, annealing, and vapor HF. The image of the wafer prior to vapor HF can be seen in Figure S4 (d), where a colorful interference gradient can be observed. By carefully tuning the process duration $(2 \mathrm{~s})$, we are able to remove most of the HSQ on the sample, while leaving the thermal oxide layer almost untouched. Thus the HSQ has a negligible contribution to the background fluorescence under laser excitation. The sample shown in Figure 3 (a) of the main text received an excess HF treatment (10 s). The zoomed in image is shown in Figure S5 (c), where an undercut region (rainbow color) with $\approx 18 \mu \mathrm{m}$ width can be seen, as well as some undesired surface patterns on the $\mathrm{SiO}_{2}$ - Si carrier wafer (dark dots). Those unwanted effects are largely suppressed by shortening the process duration to $2 \mathrm{~s}$, as seen in Figure S5 (d). The undercut width is $\approx 5 \mu \mathrm{m}$ with no visible damage on the carrier wafer.

\subsection{Dopant and isotope characterization via SIMS}

The quality of the isotopic enrichment (replacement of naturally abundant ${ }^{13} \mathrm{C}$ with ${ }^{12} \mathrm{C}$ ) is characterized via SIMS (EAG labs) and a typical carbon isotope scan is shown in Figure S9 (a). 
We reproducibly achieve 99.99 at. $\%{ }^{12} \mathrm{C}$ diamond during overgrowth, a two orders of magnitude reduction compared to natural abundance as shown in Figure S9 (a). This is currently limited only by the isotopic enrichment of the precursor $\mathrm{CH}_{4}$ gas.

Nitrogen doping effectiveness as a function of nitrogen precursor gas flow rate was also characterized via SIMS (EAG labs). Characterization of this was done via multiple scans on multiple delta doped samples with specific nitrogen doped layers (multiplicity for statistics). Fitting of the Gaussians (given the delta-doping layer thickness, a Gaussian serves as a valid approximation for the nitrogen distribution) was used to quantify the nitrogen density, which was only restricted by the depth distribution of these delta-doped layers. The FWHM is then used as an approximation of the delta-doping layer thickness, whereas the amplitude is used as an approximation of the atomic density $\left(\mathrm{cm}^{-3}\right)$ within that layer. A typical characterization run for a ${ }^{15} \mathrm{~N}$ triple delta-doped diamond is presented in Figure S9 (b). It remains to be noted that we also regularly characterize the presence of ${ }^{14} \mathrm{~N}$ to look for contamination, however, this value is regularly below the SIMS detection limit of about $2 \times 10^{-15} \mathrm{~cm}^{-3}$.

\section{3 group IV center characterization}

\subsection{Experimental setup for group IV center measurements}

The membrane samples are mounted in a closed-loop cryostation (Montana S200) for low temperature measurements. The sample temperature during the measurements is $5.38 \mathrm{~K}$ as ascertained by a sensor (Lakeshore Cryotronics) installed directly on the sample mount. The position of the membrane is controlled by three closed-loop piezo positioners (Attocube ANC 350). The incident light beams are navigated by a fast steering mirror (Newport FSM-300) controlled by an analog output device (National Instruments, PCIe-6738). For the PL measurements, we use a $532 \mathrm{~nm}$ continuous wave (CW) laser (Lighthouse Photonics Sprout-G) as the excitation source. For photoluminescence excitation (PLE) measurements of $\mathrm{GeV}^{-}$, the excitation laser is generated by a wave mixing module (AdvR Inc.) combining a tunable $\mathrm{CW}$ Ti:Sapphire laser (M Squared Solstis) and a monochromatic CW laser in telecommunication band (Thorlabs, SFL 1550P). We use this laser to scan across the $\mathrm{GeV}^{-} \mathrm{ZPL}$ wavelength (C line) and collect phonon sideband counts. At the collection ports, we use a $50: 50$ cube beam splitter (Thorlabs Inc. BS013) to divide signals to two branches for counts collection and spectroscopy measurements. A single photon counting module (SPCM) (Excelitas Technologies) is mounted to the counting beam, while a $1200 \mathrm{grmm}^{-1}$ spectrometer (Princeton Instruments, SpectraPro HRS) is fiber coupled to the spectra beam. The camera of the spectrometer (Princeton Instruments, PIXIS) is maintained at $\leq-70{ }^{\circ} \mathrm{C}$ to minimize background counts. The spectrometer branch can also be connected to another SPCM to perform autocorrelation measurements. We use a combination of bandpass filters (Semrock FF01-615/24-25, Semrock FF01-600/14-25) for $\mathrm{GeV}^{-}$PL measurements. When performing PLE measurements, we collected the phonon sidebands via double filters (2× Semrock FF01-647/57-25). All lenses in 
the confocal setup are achromatic doublets with AB coatings (Thorlabs Inc.) to reach maximum transmission efficiency over broadbands. The PL map obtains a very low background fluorescence $(\approx 1500 \mathrm{~Hz}$ ) even under $10 \mathrm{~mW}$ excitation, as shown in Figure 3 (b) of the main text. The optical setups for $\mathrm{SiV}^{-}$and $\mathrm{SnV}^{-}$characterizations are almost identical, except for the bandpass filter (Semrock FF01-740/13-25 for $\mathrm{SiV}^{-}$and a Semrock FF01-615/24-25 for $\mathrm{SnV}^{-}$). Since $\mathrm{GeV}^{-}$centers are also present in the PL map of $\mathrm{SnV}^{-}$centers, we use the spectrometer to identify the center type before proceeding with further characterizations.

\section{2 $\mathrm{GeV}^{-}$creation efficiency}

We estimate the creation yield of $\mathrm{GeV}^{-}$by counting the number of fluorescent centers in a certain area. Given the good signal-to-background ratio of $\approx 30$, any resolvable centers when counting each $20 \mu \mathrm{m}$ by $20 \mu \mathrm{m}$ PL map were included in our counting. The average number of centers per areal spot is $53(3)\left(\approx 0.13 \mu \mathrm{m}^{-2}\right)$, yielding a creation yield of $6.6(4) \%$ from the implantation dose $\left(2 \times 10^{8} \mathrm{~cm}^{-2} \approx 2 \mu \mathrm{m}^{-2}\right)$. This yield is higher than the previous reported value ${ }^{17}$. We note the high signal-to-background ratio can improve the visibility of darker $\mathrm{GeV}^{-}$centers, which potentially increase the estimated yield.

\subsection{Spectra of Group IV centers and GeV auto-correlation measurements}

The $\mathrm{GeV}^{-}$membrane sample were co-doped with $\mathrm{Si}^{+}$and $\mathrm{Sn}^{+}$with the same density and target depth. Figure S6 (a-c) shows the representative spectra of $\mathrm{GeV}^{-}, \mathrm{SiV}^{-}$and $\mathrm{SnV}^{-}$centers under $5.4 \mathrm{~K}$. Like $\mathrm{GeV}^{-}$centers, the ZPL peaks of $\mathrm{SiV}^{-}$and $\mathrm{SnV}^{-}$are comparable with the bulk diamond values ${ }^{18,19}$. The $\mathrm{A}$ and $\mathrm{B}$ peaks of $\mathrm{SnV}^{-}$are not resolvable due to large spin-orbit coupling and limited phonon population under such a low temperature. More in-depth studies of $\mathrm{SiV}^{-}$and $\mathrm{SnV}^{-}$centers are left for subsequent work to explore.

Off- and on-resonance measurements are performed on $\mathrm{GeV}^{-}$centers, as shown in Figure S6 (d). Both curves exhibit anti-bunching features with $g^{(2)}(0)$ well below 0.5 , originated from their single center nature. During PL auto-correlation measurements, the off-resonance laser $(532 \mathrm{~nm})$ is operated at a slightly lower power $(3 \mathrm{~mW})$ to avoid bunching effects. The PLE autocorrelation measurement indicates a clear Rabi oscillation pattern induced by an onresonance excitation.

The reasons for the non-zero $g^{(2)}(0)$ values $(0.17(2)$ for PL and $0.19(5)$ for PLE) depend on the specific type of measurements. For PL autorrelation measurements, we observed a slowly decreasing ZPL signal with respect to the measurement time. This can be explained by a gradually changing surface termination with the presence of the excitation laser, which affects the preferred electronic configuration of $\mathrm{GeV}^{-}$centers. As a result, the color centers may experience more times in their optically dark state, slowly quenching the overall signal. In the future, a more rigorous surface termination scheme, such as a tri-acid clean or oxygen 
termination can be applied to improve the $\mathrm{GeV}^{-}$stability. For the PLE autocorrelation measurements, the average ZPL linewidth is $\approx 2.4$ times wider than the single scan values. With the frequency of the excitation source fixed, the spectral diffusion causes considerable fluctuations of phonon sideband counts, raising the PLE $g^{(2)}(0)$ value. Future optimizations on the linewidth of the $\mathrm{GeV}^{-}$emissions can effectively enhance the average signal and suppress the $g^{(2)}(0)$.

\section{4 $\mathrm{GeV}^{-}$emission linewidth and spectral jump analysis}

The optical linewidth of $\mathrm{GeV}^{-}$centers approaches the lifetime limit by a factor of 3 (6) during single (average) scans. While the synthesis of optimized narrow linewidth color centers is beyond the scope of this work, multiple strategies can be introduced to overcome the extrinsic broadening factors. To begin with, the optical coherence is impacted by thermal effects via electron-phonon interactions, as modeled and characterized $\mathrm{in}^{20}$. Therefore, narrower linewidths may be achievable at lower characterization temperatures. Additionally, some inhomogeneous broadening is attributed to the implantation-induced damage ${ }^{21}$. In theory, this can be minimized by doping Ge in-situ during the overgrowth process ${ }^{22}$, which is left for subsequent studies to explore. Furthermore, optimized surface terminations should contribute to reducing surface charge related noise. We excluded the optical power broadening in this work by characterizing the saturation power and tuning the excitation to be slightly lower than this saturation point before every linewidth measurement.

We also observed the switching phenomena for $\mathrm{GeV}^{-}$in most membrane samples during PLE measurements. A long term scan (25 min) of a single $\mathrm{GeV}^{-}$is shown in Figure S6 (e). This center is located in a $20 \mathrm{~h}$ membrane sample with only $\mathrm{Si}^{+}$and $\mathrm{Ge}^{+}$implantation. The origin of the switching is currently under investigation.

\section{5 $\mathrm{GeV}^{-}$zero phonon lines distribution}

To evaluate the inhomogeneous broadening of the implanted $\mathrm{GeV}^{-}$centers, we included an electronic grade diamond as a reference sample during the $\mathrm{Ge}^{+}$implantation and subsequent annealing. Therefore, the reference diamond received identical ion implantation and annealing steps. Under the same cryostation temperature $(5.4 \mathrm{~K})$, we measured a total of $75 \mathrm{GeV}^{-}$centers in diamond membranes and 30 centers in the reference sample, shown in Figure S6 (f) left and right. If all centers are included in deriving the inhomogeneous broadening, we find a standard deviation of $\sigma=1.16 \mathrm{~nm}$ for the membrane and $\sigma=1.52 \mathrm{~nm}$ for the reference sample. However, these numbers are mostly influenced by $\approx 10 \%$ of the centers with large wavelength shifts (such as $607 \mathrm{~nm}$ ). These shifts are possibly induced by local strain distortion from the implantation process, and can be found in both membrane and bulk cases. Moreover,these $\sigma$ values are expected to have a large uncertainty due to limited sampling. Following a similar analysis ${ }^{23}$, confining the ZPL wavelengths of $\mathrm{GeV}^{-}$centers to within a range between $601.5 \mathrm{~nm}$ and 603.2 $\mathrm{nm}$ (which includes $\approx 90 \%$ of the centers) may give a better value for comparison. Compared to 
the inhomogeneous broadening in the reference $(\sigma=0.18 \mathrm{~nm})$, the values we obtained are $\sigma=$ $0.18 \mathrm{~nm}$ for the reference diamond and $\sigma=0.19 \mathrm{~nm}$ for the diamond membranes. These similar inhomogeneous broadening values indicate comparable crystal environments between the diamond membrane and bulk diamond.

\section{$4 \quad \mathrm{NV}^{-}$characterization}

\subsection{Experimental setup for $\mathbf{N V}^{-}$characterization}

The room temperature $\mathrm{NV}^{-}$coherence measurements were performed in a custom-built confocal microscope with a $532 \mathrm{~nm}$, free-space laser (Oxxius 532S-150-COL-PP) and a 100X, 0.9NA air objective (Olympus MPLFLN100X). The laser spot position is controlled with a fast steering mirror (Newport FSM-300) and a 4f lens pair. The laser is gated with an acousto-optic modulator (AOM) (Gooch \& Housego) and PL is measured with a PDM photon counter (MPD PD-050-CTD-FC). Microwave pulses are generated by a SRS SG-396, amplified by a MiniCircuits ZHL-15W-422-S+, and gated by both a Mini-Circuits ZASWA-2-50DR+ RF switch and external amplitude modulation of the signal generator. Pulses are applied to the $\mathrm{NV}^{-}$ centers via a $25 \mu \mathrm{m}$ Al wire draped over the sample. The experiment timing is handled by a Swabian Pulse Streamer.

A static magnetic field of 15 gauss is applied at approximately $35^{\circ}$ to the [100] sample surface. Given the orientation of the sample, the field is at a $10^{\circ}$ angle to the [111] crystal axis.

\section{2 $\mathrm{NV}^{-}$coherence measurements and $\mathrm{T}_{2}$ comparison with delta-doped results from previous studies}

We use standard free induction decay (FID) and spin echo (SE) sequences to measure $T_{2}^{*}$ and $T_{2}$, respectively. Each sequence, shown in Figure S8, is composed of two branches, which measure both the $|0\rangle$ and $|1\rangle$ projections. After applying a $\pi / 2$-pulse on the $\mathrm{NV}^{-}$, we allow the spin to evolve for a time $\tau$ (and apply a $\pi_{y}$ pulse at $\tau / 2$ for the SE) before applying either another $+\pi / 2$-pulse or a $-\pi / 2$-pulse to project into the readout basis. The readout windows at the beginning of each laser pulse are binned as the readout signals, $S_{0,-1}$, and the readout window at the end is binned as a reference, $R$, which allows us to correct for slow drift over each measurement cycle ${ }^{24}$. All measurements are processed as

$$
\text { Coherence }=\frac{\mathrm{S}_{0}-\mathrm{S}_{-1}}{\mathrm{R}} \cdot \frac{\mathrm{R}[0]}{\mathrm{S}_{0}[0]+\mathrm{S}_{-1}[0]},
$$

where the second term accounts for absolute count discrepancies between readout at the beginning and end of the laser pulse that arise from the finite rise time of the AOM. Each pulse 
sequence is a different length due to sweeping $\tau$, but run for the same amount of time overall, necessitating normalization by the reference to cancel this variation. Ultimately this means that shorter- $\tau$ data are average over more instances than longer- $\tau$ data. We fit $T_{2}^{*}$ measurements to the following 25

$$
B+\exp \left[-\left(t / T 2^{*}\right)^{2}\right] \cdot \sum_{i=1}^{2 I+1} a_{i} \cos \left(2 \pi f_{i}\left(t+\phi_{i}\right)\right),
$$

Where $B$ is a background offset, $a_{i}, f_{i}$, and $\varphi_{i}$ are the amplitude, frequency, and phase of each sinusoid corresponding to each hyperfine peak, and $I$ is the nitrogen nuclear spin $\left(1 / 2\right.$ for ${ }^{15} \mathrm{~N}$, 1 for $\left.{ }^{14} \mathrm{~N}\right) . T_{2}$ measurements are fit to

$$
B+A \exp \left[-\left(t / T_{2}\right)^{n}\right]
$$

Where $B$ is an offset, $A$ is the amplitude, and $n$ is either a free parameter or fixed at $3^{25}$, when the former does not fit.

Previous delta-doping work demonstrated $T_{2}$ times for deep $\mathrm{NV}^{-}$centers $(\geq 52 \mathrm{~nm})$ on the order of $\approx 700 \mu \mathrm{s}^{3}$, with measured values dropping drastically $(\leq 100 \mu \mathrm{s})$ as the delta-doped layer was brought close to the diamond surface $(\leq 35 \mathrm{~nm})$. Indeed, it is expected that surfacerelated decoherence is a main limitation for the $T_{2}$ times presented in this work ${ }^{12}$. The impact of surfaces is further amplified in membranes, as the color centers are in close proximity to two surfaces rather than one.

\section{3 $\quad \mathrm{NV}^{-}$strain measurements}

The $\mathrm{NV}^{-}$center is sensitive to both strain and electric field (I) through spin state coupling, as shown in the Hamiltonian

$$
H=\left(D+\Pi_{z}\right) S_{z}^{2}+\Pi_{x}\left(S_{y}^{2}-S_{x}^{2}\right)+\Pi_{y}\left(S_{x} S_{y}+S_{y} S_{x}\right) .
$$

Parallel strain/electric field $\left(\Pi_{z}\right)$ shifts the effective zero-field splitting, while the perpendicular components $\left(\Pi_{x, y}\right)$ split the upper and lower spin transitions ${ }^{26}$. However, there is a discrepancy in the strength of the couplings for each physical origin. The electric field susceptibilities $d_{\epsilon: \|, \perp}=0.35 \mathrm{HzcmV}^{-1}$ and $17 \mathrm{HzcmV}^{-127}$, while the strain susceptibilities $d_{\sigma: \mathrm{k}, \perp}$ $=13.3 \mathrm{GHz}$ and $21.5 \mathrm{GHz}^{28}$. We thus expect that if local electric fields dominate, the splitting between the upper and lower transitions should be much larger than the shifts in zero-field splitting, whereas if strain dominates these effects should be of similar magnitudes.

Through zero-field ODMR measurements, we found in all but one case that the splitting was greater than or the same magnitude as the shifts. In Figure S7 (a) we present the shifts in zerofield splitting versus the NV orientation, as determined at non-zero field, to extract an upper bound on the strain in the membrane. If we assume the larger shifts of $\approx 1 \mathrm{MHz}$ arise entirely from strain - an over-estimate, given the above discussion - we arrive at a strain of $<10^{-4}$. 
Multiple factors can contribute to the built-in strain. For instance, the dry transfer step might introduce in-plane strain due to approach angle and speed. This can be reduced by reducing the approaching speed of the PDMS stamp with a smaller angle. Additionally, although the HSQ layer collapses above $465{ }^{\circ} \mathrm{C}$ and potentially releases the strain during the annealing, some residual strain might still remain. Furthermore, due to different thermal expansion ratios between the diamond membrane and carrier wafers, a thermal-induced strain appears when the sample is cooled down to room temperature or at $5.4 \mathrm{~K}$ of PL characterizations. The direction and the magnitude of the strain depend on the material of the carrier wafers. Finally, some onchip structures (such as trenches) can alter the strain distribution across the membrane.

\section{4 $\mathrm{NV}^{-} \boldsymbol{T}_{1}$ comparison}

We compare $T_{1}$ population decay times for two $\mathrm{NV}^{-}$centers with an order of magnitude separation in coherence times to confirm that a reduction in $T_{1}$ is not responsible for the reduction in $T_{2}$. Figure $\mathrm{S} 7(\mathrm{~b})$ shows the differential $T_{1}$ measurement for NVX $\left(T_{2}=13(1) \mu \mathrm{s}\right)$ and NVY $\left(T_{2}=146(53) \mu \mathrm{s}\right)$. Over $5 \mathrm{~ms}$ there is no observable increased decay for NVX, indicating $T_{1}$ plays no role in limiting coherence.

\section{$4.5[\mathrm{~N}]$ and $\left[\mathrm{NV}^{-}\right]$estimation}

Both the overgrowth background $\left[{ }^{14} \mathrm{~N}\right]$ and in-situ doped $\left[{ }^{15} \mathrm{~N}\right]$ were determined via calibrated quantitative secondary ion mass spectroscopy (SIMS) characterization (EAG Laboratories). All SIMS derived error bars are standard deviation from a duplicate experimental characterization. The SIMS detection limit of $\left[{ }^{14} \mathrm{~N}\right]$ placed an upper bound on the background nitrogen contamination $4.5(2) \times 10^{15} \mathrm{~cm}^{-3}(\leq 26(1) \mathrm{ppb})$. For the $0.06 \mathrm{sccm}{ }^{15} \mathrm{~N}_{2}$ dopant precursor flow rate used for the generation of in-situ doped $\delta$-doped membranes, we obtain a $\left[{ }^{15} \mathrm{~N}\right]$ of $5.1(10) \times 10^{15} \mathrm{~cm}^{-3}(30.8(57) \mathrm{ppb})$ with an EAG quoted ${ }^{15} \mathrm{~N}$ detection limit of $1 \times 10^{15} \mathrm{~cm}^{-3}(5.7$ $\mathrm{ppb})$.

The nitrogen implanted membrane sample was subjected to a $48 \mathrm{keV}, 2 \times 10^{8} \mathrm{~cm}^{-2}[\mathrm{~N}]$ dose. At this energy, the straggle (standard deviation of implantation as approximated with SRIM) is $14 \mathrm{~nm}$. Assuming the bulk of the nitrogen resides in a $\leq 30 \mathrm{~nm}$ thick region, the expected [N] from the implantation to be $6.7 \times 10^{13} \mathrm{~cm}^{-3}(\approx 0.4 \mathrm{ppb})$ within the implanted region. Comparing this to the observed $\left[{ }^{14} \mathrm{NV}^{-}\right]$of $1.8 \times 10^{12} \mathrm{~cm}^{-3}(0.01 \mathrm{ppb})$ obtained from a typical count of a 10 $\mu \mathrm{m}$ by $10 \mu \mathrm{m}$ region of the $120 \mathrm{~nm}$ thick membrane. This suggests an upper bound on the N-to$\mathrm{NV}$ conversion efficiency at $\leq 2.5 \%$. Realistically, in-grown background $[\mathrm{N}]$ are also being converted to $\left[\mathrm{NV}^{-}\right]$, causing an overestimation of this conversion efficiency.

Isotopic tagging of dopant species in the ${ }^{15} \mathrm{~N} \delta$-doped membrane was used to provide further insight into this. From a PL survey of a $12 \mu \mathrm{m}$ by $12 \mu \mathrm{m}$ area of the $110 \mathrm{~nm}$ thick delta-doped membrane (post-irradiation and annealing), we noted a relative ratio of $7: 11$ for $\left[{ }^{15} \mathrm{NV}^{-}\right]$: $\left[{ }^{14} \mathrm{NV}^{-}\right]$. Knowing that the delta doped area is $\approx 2 \mathrm{~nm}$ thick, with $\left[{ }^{15} \mathrm{~N}\right]=5.1(10) \times 10^{15} \mathrm{~cm}^{-3}$, we 
can work out the approximate density of the $\left[{ }^{14} \mathrm{~N}\right]$ in the rest of the membrane-assuming a uniform conversion efficiency for both the $\delta$-doped $\left[{ }^{15} \mathrm{~N}\right]$ and background in-grown natural abundance $[\mathrm{N}]$ (this approximation can break down easily, but remains useful here). Once geometric effective layer thicknesses are considered, i.e., $2 \mathrm{~nm}$ thick portion of $\left[{ }^{15} \mathrm{~N}\right]$ vs a 110 $\mathrm{nm}$ thick $\approx\left[{ }^{14} \mathrm{~N}\right]$ membrane, we end up with a normalized observed $\left[{ }^{15} \mathrm{NV}^{-}\right]:\left[{ }^{14} \mathrm{NV}^{-}\right]$normalized of $7: 0.2$, resulting in a background $\left[{ }^{14} \mathrm{~N}\right]$ of $0.124(5) \times 10^{15} \mathrm{~cm}^{-3}(0.70(3) \mathrm{ppb})$.

\section{References}

[1] B. C. Rose, et al., Science 361, 60 (2018).

[2] P. John, M. D. Stoikou, Physical Chemistry Chemical Physics 13, 11503 (2011).

[3] K. Ohno, et al., Applied Physics Letters 101 (2012).

[4] S. A. Meynell, et al., Applied Physics Letters 117, 194001 (2020).

[5] N. Yang, ed., Novel Aspects of Diamond, vol. 121 of Topics in Applied Physics (Springer International Publishing, Cham, 2015).

[6] J. C. Lee, A. P. Magyar, D. O. Bracher, I. Aharonovich, E. L. Hu, Diamond and Related Materials 33, 45 (2013).

[7] M. Ruf, et al., Nano Letters 19, 3987 (2019).

[8] J. F. Ziegler, M. D. Ziegler, J. P. Biersack, Nuclear Inst. and Methods in Physics Research, B 268, 1818 (2010).

[9] K. J. Brown, E. Chartier, E. M. Sweet, D. A. Hopper, L. C. Bassett, ACS Chemical Health \& Safety (2021).

[10] K. M. Fu, C. Santori, P. E. Barclay, R. G. Beausoleil, Applied Physics Letters 96, 1 (2010).

[11] A. H. Piracha, et al., Nano Letters 16, 3341 (2016).

[12] S. Sangtawesin, et al., Physical Review X 9, 1 (2019).

[13] A. C. Ferrari, Solid State Communications 143, 47 (2007).

[14] A. P. Magyar, et al., Applied Physics Letters 99, 1 (2011).

[15] A. Butcher, et al., Nano Letters 20, 4603 (2020).

[16] M. N. Polyanskiy, Refractive index database, https://refractiveindex.info. 
[17] N. H. Wan, et al., Nature 583, 226 (2020).

[18] S. Meesala, et al., Physical Review B 97, 1 (2018).

[19] M. E. Trusheim, et al., Physical Review Letters 124, 1 (2020).

[20] K. D. Jahnke, et al., New Journal of Physics 17 (2015).

[21] S. B. Van Dam, et al., Physical Review B 99, 161203 (2019).

[22] A. Sipahigil, et al., Physical Review Letters 113, 1 (2014).

[23] T. Iwasaki, et al., Scientific Reports 5, 1 (2015).

[24] B. A. Myers, A. Ariyaratne, A. C. Jayich, Physical Review Letters 118, 1 (2017).

[25] L. Childress, et al., Science 314, 281 (2006).

[26] T. Mittiga, et al., Phys. Rev. Lett. 121, 246402 (2018).

[27] E. Van Oort, M. Glasbeek, Chemical Physics Letters 168, 529 (1990).

[28] P. Ovartchaiyapong, K. W. Lee, B. A. Myers, A. C. B. Jayich, Nature Communications 5, 4429 (2014). 


\begin{tabular}{|c|cccc|}
\hline Species & Energy [keV] & Dose $\left[\mathrm{cm}^{-2}\right]$ & Target depth [nm] & Straggle [nm] \\
\hline${ }^{4} \mathrm{He}^{+}$ & 150 & $5 \times 10^{16}$ & 413 & 39 \\
\hline${ }^{14} \mathrm{~N}^{+}$ & 48 & $2 \times 10^{8}$ & 60 & 15 \\
\hline${ }^{28} \mathrm{Si}^{+}$ & 58 & $2 \times 10^{8}$ & 40 & 11 \\
\hline${ }^{74} \mathrm{Ge}^{+}$ & 98 & $2 \times 10^{8}$ & 40 & 10 \\
\hline${ }^{120} \mathrm{Sn}^{+}$ & 150 & $2 \times 10^{8}$ & 42 & 8 \\
\hline
\end{tabular}

Table S1: Implantation parameters and SRIM simulation results for diamond membrane formation and color center generation.

\begin{tabular}{|c|c|c|c|c|}
\hline Recipe name & $\mathrm{Ar} / \mathrm{Cl}_{2}$ & $\mathrm{O}_{2} / \mathrm{Cl}_{2}$ & $\mathrm{O}_{2}$ & $\mathrm{Al}_{2} \mathrm{O}_{3}$ etching \\
\hline ICP power $[\mathrm{W}]$ & \multirow{7}{*}{$\begin{array}{c}400 \\
250 \\
8 \\
40 \\
25 \\
0 \\
0\end{array}$} & \multirow{7}{*}{$\begin{array}{c}700 \\
100 \\
10 \\
2 \\
0 \\
0 \\
30\end{array}$} & \multirow{7}{*}{$\begin{array}{c}700 \\
100 \\
10 \\
0 \\
0 \\
0 \\
30\end{array}$} & \multirow{7}{*}{$\begin{array}{c}400 \\
50 \\
5 \\
0 \\
10 \\
30 \\
0\end{array}$} \\
\hline Bias power [W] & & & & \\
\hline Pressure [mTorr] & & & & \\
\hline $\mathrm{Cl}_{2}$ flow $[\mathrm{sccm}]$ & & & & \\
\hline Ar flow [sccm] & & & & \\
\hline $\mathrm{BCl}_{3}$ flow $[\mathrm{sccm}]$ & & & & \\
\hline $\mathrm{O}_{2}$ flow $[\mathrm{sccm}]$ & & & & \\
\hline Etching rate $\left[\mathrm{nmmin}^{-1}\right]$ & $\approx 73$ & $\approx 177$ & $\approx 175$ & $\approx 63\left(\mathrm{Al}_{2} \mathrm{O}_{3}\right)$ \\
\hline
\end{tabular}

Table S2: Cl-based ICP etching recipe for diamond. The last recipe, $\mathrm{Al}_{2} \mathrm{O}_{3}$ etching, is used for hard mask $\left(\mathrm{ALD} \mathrm{Al}_{2} \mathrm{O}_{3}\right)$ removal during the membrane patterning. 

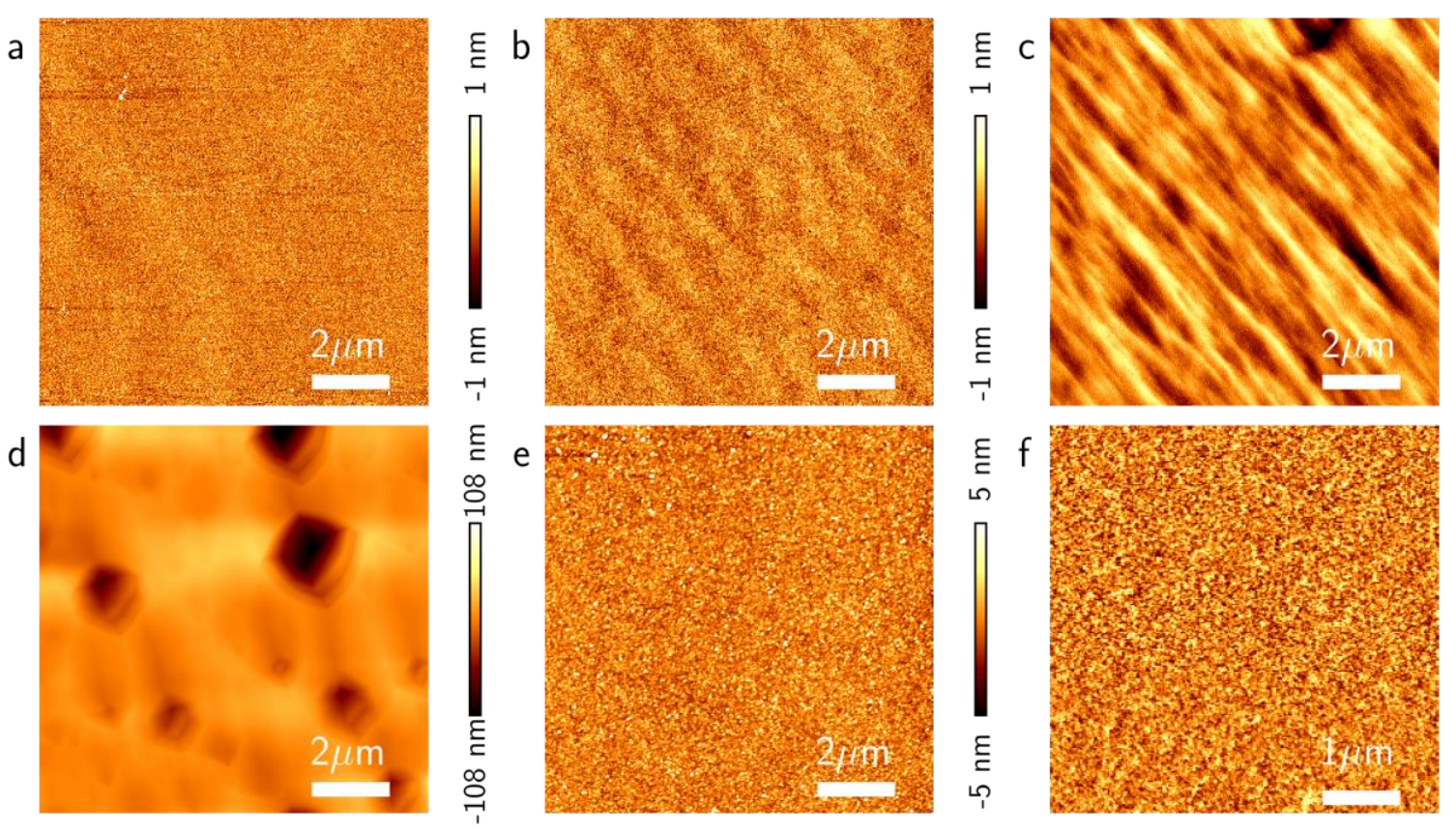

Figure S1: AFM characterizations during diamond membrane synthesis. (a) Fine polished diamond substrate shows $R q=0.30 \mathrm{~nm}$. (b) The substrate after implantation and annealing with $R q=0.27 \mathrm{~nm}$. (c) The 40h overgrown sample with 60min hydrogen etch. $R q=0.69 \mathrm{~nm}$. (d) The 40 h overgrown sample with insufficient surface cleaning prior to the growth. $R q=18.6 \mathrm{~nm}$. (e) Backside of the membrane after the dry transfer. $R q=1.17 \mathrm{~nm}$. (f) Backside of the membrane after $30 \mathrm{~s}$ of oxygen ICP with no prior " $\mathrm{Ar} / \mathrm{Cl}_{2}$ " etching. $R q=6.78 \mathrm{~nm}$. 

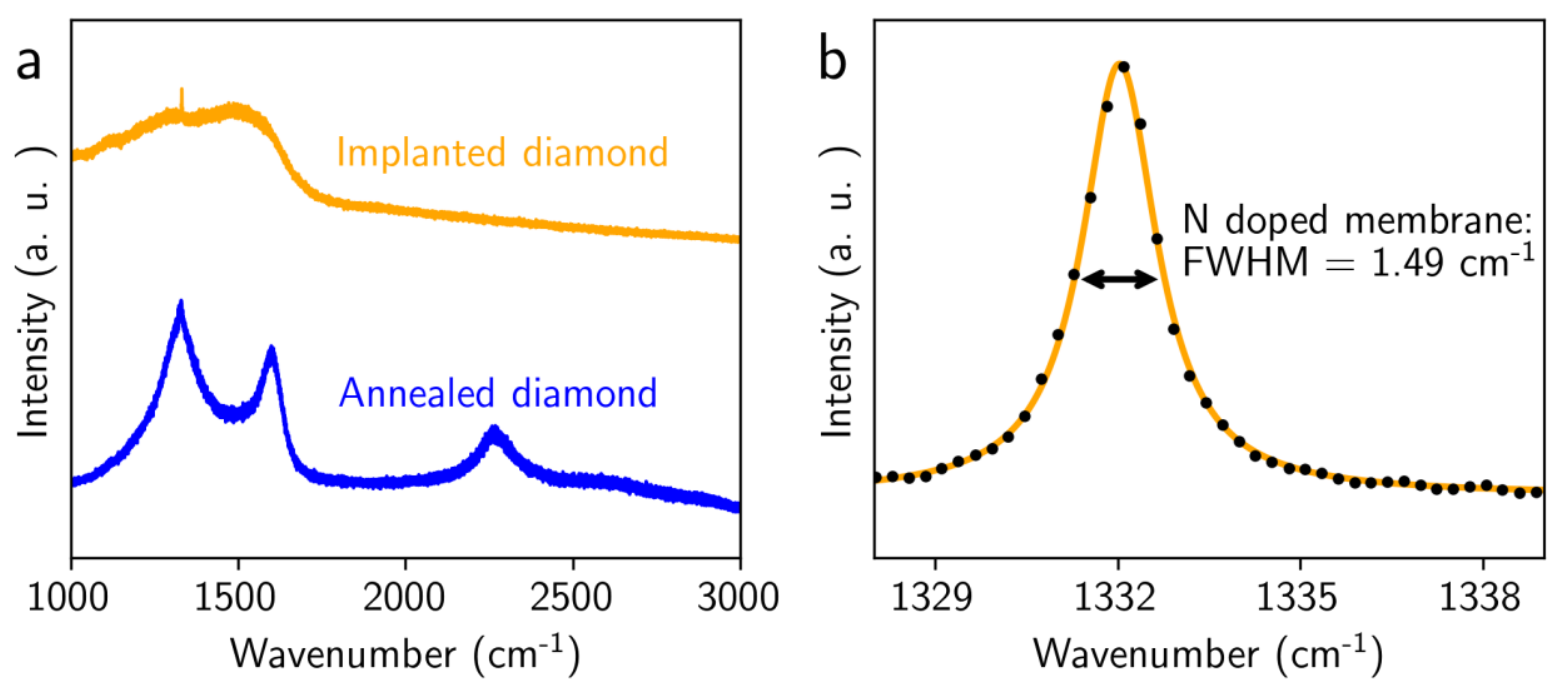

Figure S2: Additional Raman spectroscopy for membrane formation and overgrowth. (a) Orange curve: the implanted diamond substrate prior to annealing. Blue curve: the annealed diamond substrate. (b) The Raman peak for an isotopically purified, $\approx 250 \mathrm{~nm}$ overgrown membrane at $700{ }^{\circ} \mathrm{C}$ (40 h growth, back-etched down to $110 \mathrm{~nm}$ ). The peak is at $1332.03(30)$ $\mathrm{cm}^{-1}$, with linewidth $1.486(14) \mathrm{cm}^{-1}$. 

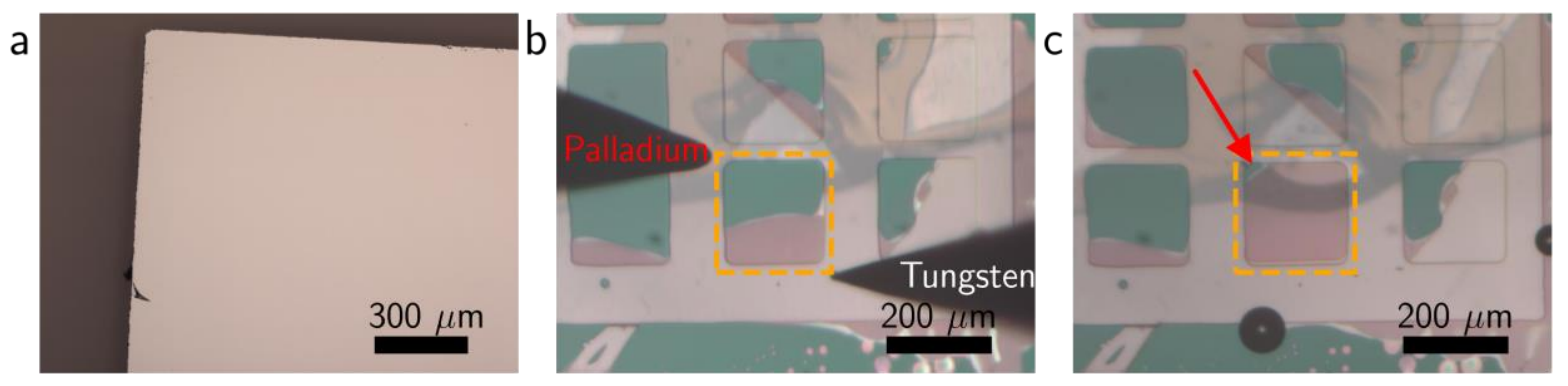

Figure S3: Microscope images for annealed and undercut diamond membranes. (a) The diamond membrane after $\mathrm{He}^{+}$implantation and subsequent annealing. The sample exhibits uniform light pink reflections, which can be interpreted as interference effect between a transparent top surface and a reflective graphitic underlayer. (b-c) A half- (almost-) undercut membrane (dashed orange square) at the EC etching step. Both images were taken when the membrane was in DI water. The left (right) electrode in (c) represents the palladium (tungsten) tip. The red arrow in (d) points to the membrane tether. The opaque graphitic layer exhibits interference colors (green), while the undercut region is almost transparent. The plate on the upper right indicates a leftover tether where the membrane has been picked up, while the membranes on the top and right were by-caught during the transfer. Such by-caught phenomena were caused by undesired EC etching due to low resistance of tap water and has been largely suppressed since the DI water is applied instead (See the membrane on the left in both images). 

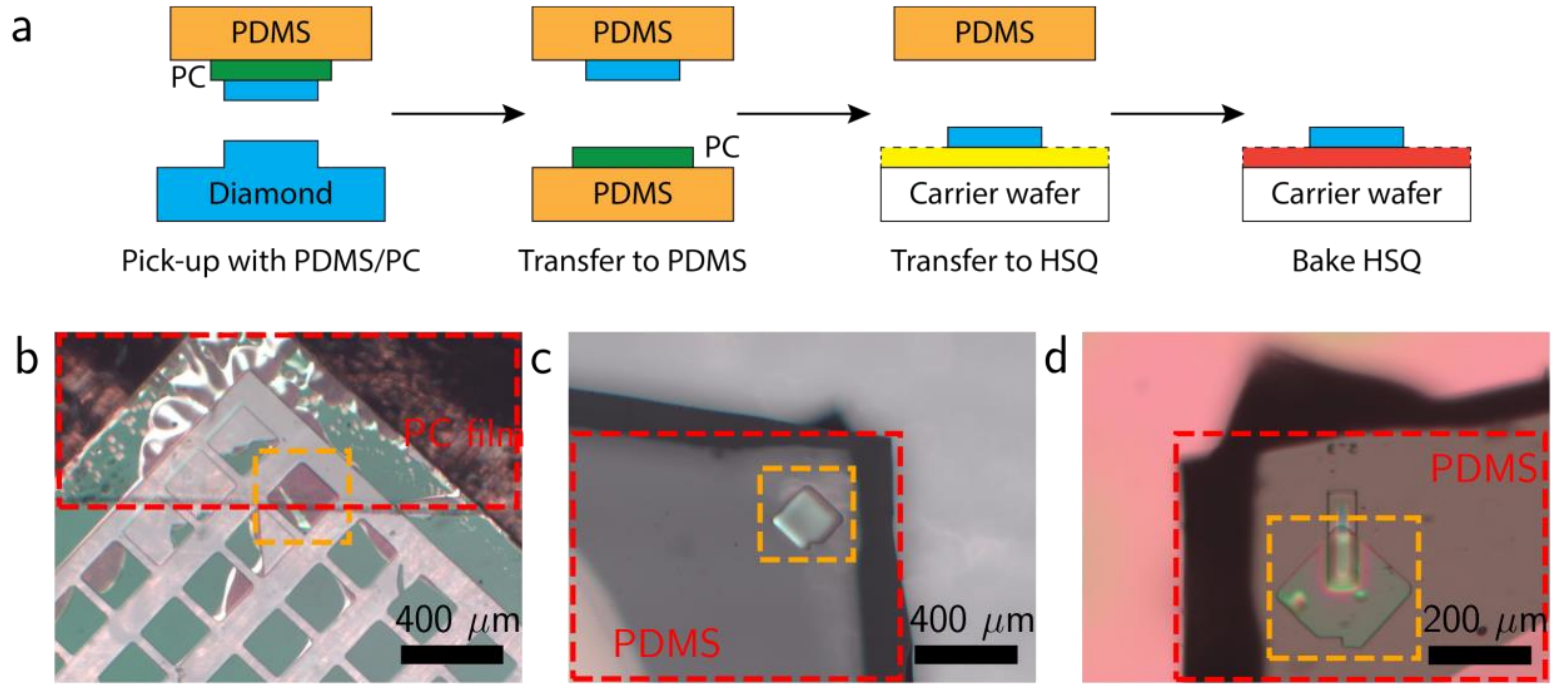

Figure S4: Deterministic diamond membrane transfer. (a) General transfer process flow. The diamond is flipped during the process in order to remove the original membrane afterwards. (b) The PDMS/PC stamp approaches the diamond membrane. The PC film (red dashed rectangle) is located under a PDMS stamp which spans beyond this image. The target membrane (orange dashed square) is linked to the diamond substrate via a small tether (See S3 (c)). (c) The membrane transferred to the PDMS-only stamp (red dashed rectangle) in the second step. (d) The membrane attached to a HSQ-coated trench on a thermal oxide wafer. The bumps under the membrane can be avoided by cleaner PDMS/PC stamp preparation in the future. 
a

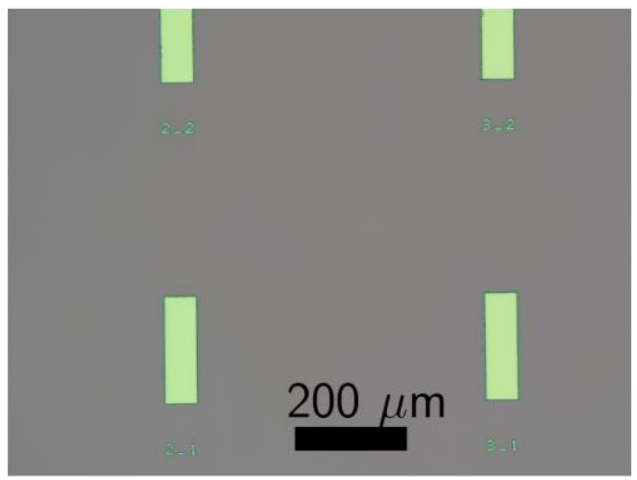

C
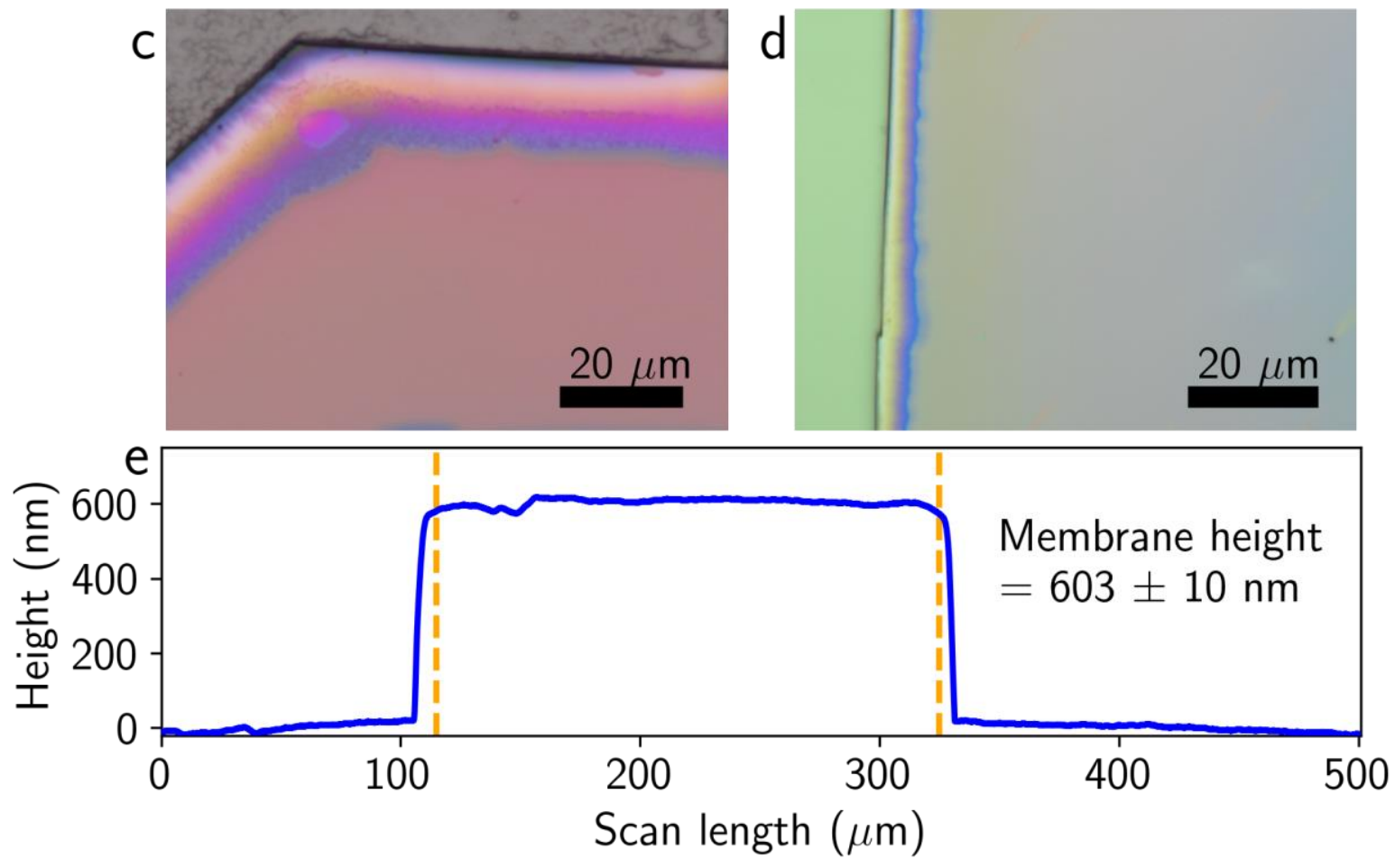

Figure S5: Carrier wafer preparation, vapor HF treatment and membrane thickness characterization. (a) $280 \mathrm{~nm} \mathrm{SiO}{ }_{2} \_$Si thermal oxide wafer with $5 \mu \mathrm{m}$ deep trenches and markers. The silicon (green) exhibits different color from the oxide layer (gray). (b) A trench on the thermal oxide wafer after HSQ spinning, annealing and vapor HF treatment. The HSQ is mostly removed while the thermal oxide layer is barely untouched. (c-d) The undercut edge of the diamond membrane after $10 \mathrm{~s}(2 \mathrm{~s})$ of vapor HF etching. (e) Profilometry characterization of the membrane thickness after transfer and before back etching. The region of the transferred membrane is indicated by the dashed orange lines. The average thickness for this ${ }^{15} \mathrm{~N}$-doped membrane is $603 \mathrm{~nm}$ with a standard deviation of $10 \mathrm{~nm}$. 

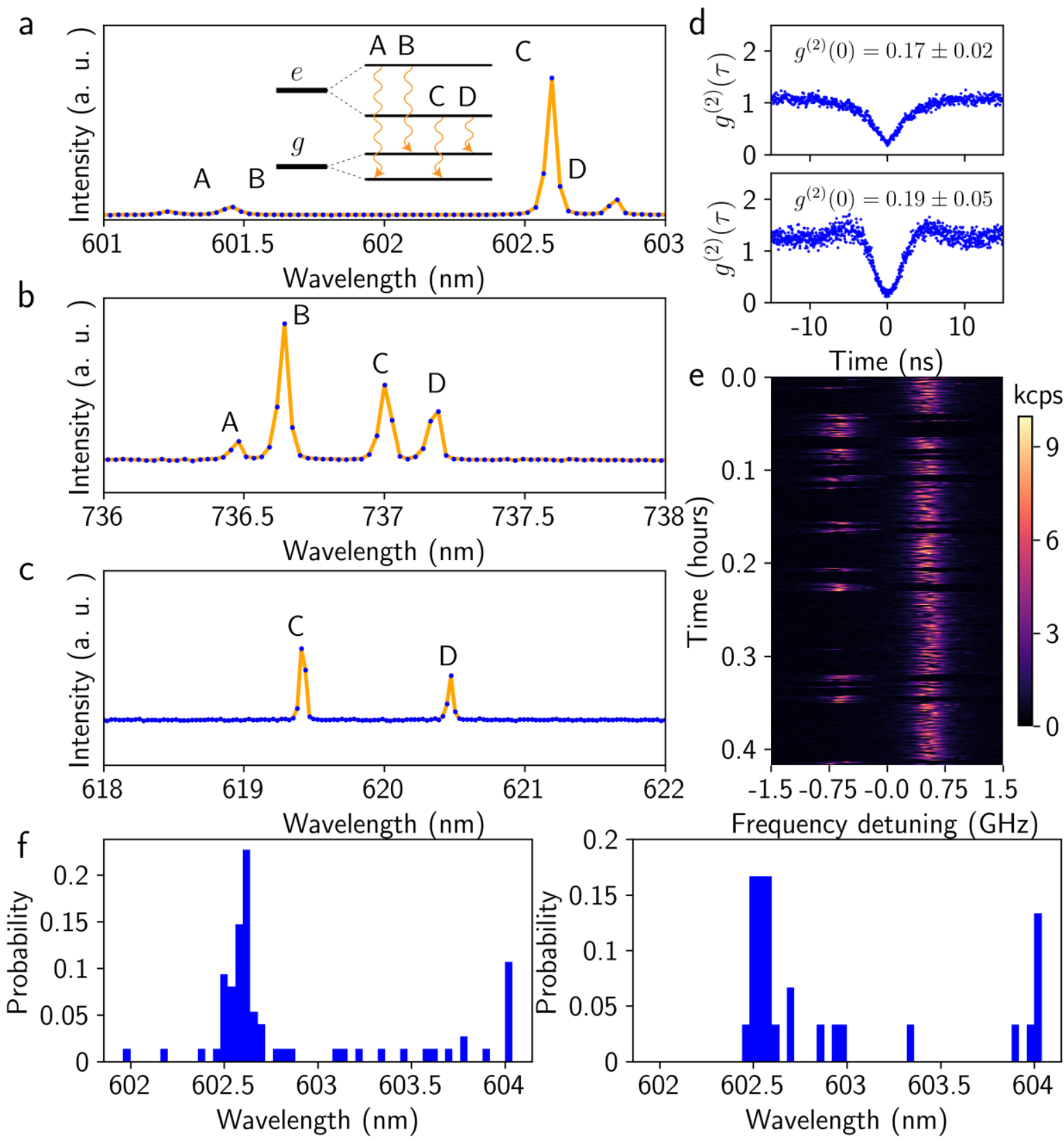

Figure S6: Additional optical measurements of group IV centers. (a-c) The spectra of a representative $\mathrm{GeV}\left(\mathrm{SiV}^{-}, \mathrm{SnV}^{-}\right)$center measured under $5.4 \mathrm{~K}$, with $10 \mathrm{~mW} 532 \mathrm{~nm}$ laser excitation. The small plot inside (a) represents the general energy levels of negatively charged group IV centers. (d) Off- (on)-resonance autocorrelation measurements of a GeV center. The excitation power of the off-resonance laser is $3 \mathrm{~mW}$, while the on-resonance laser is $40 \mathrm{nW}$. (e) A 25 min scanning of a spectrally switching $\mathrm{GeV}^{-}$center. (f) $\mathrm{ZPL}$ distributions of $\mathrm{GeV}^{-}$centers in the diamond membrane (left) and bulk diamond (right). 
a

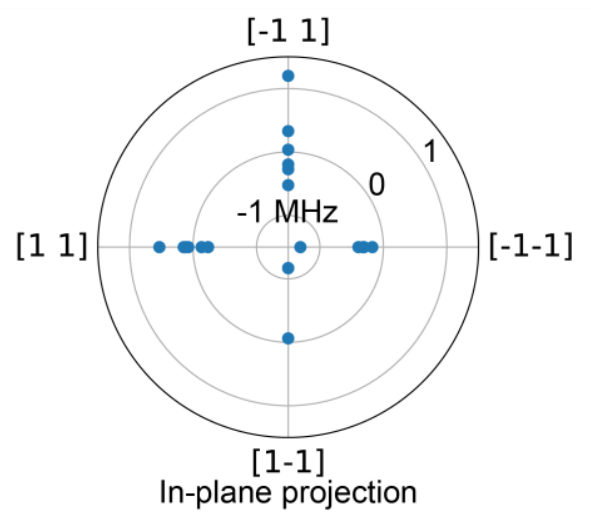

b

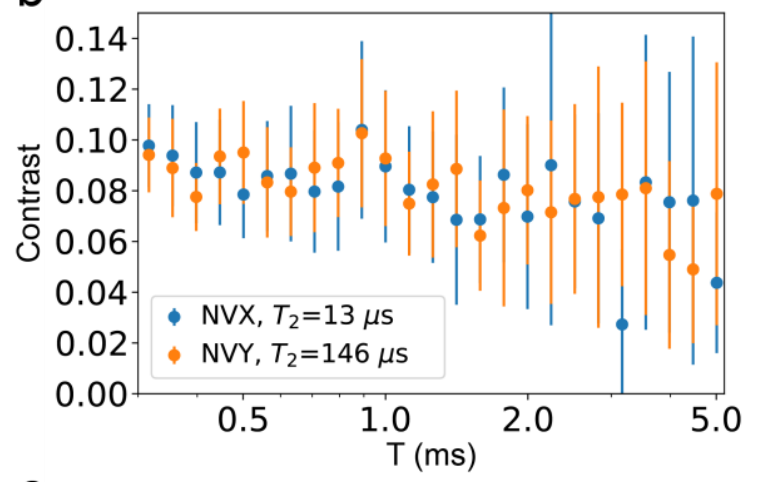

C

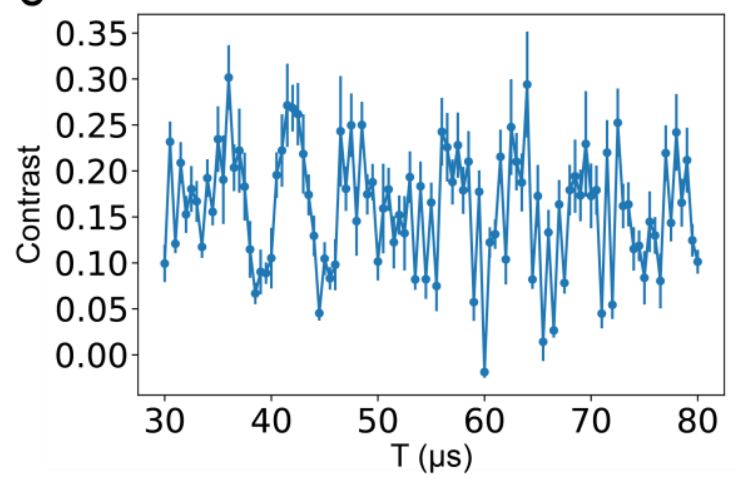

Figure S7: (a) Zero-field splitting of measured $\mathrm{NV}^{-}$centers. (b) $T_{1}$ measurement of two $\mathrm{NV}^{-} \mathrm{S}$ with order of magnitude spread in $T_{2}$ times. (c) Finer resolution spin echo measurement on the $\mathrm{NV}^{-}$presented in Fig. 4 (a), showing electron spin echo envelope modulation, which is aliased in the main text data. 


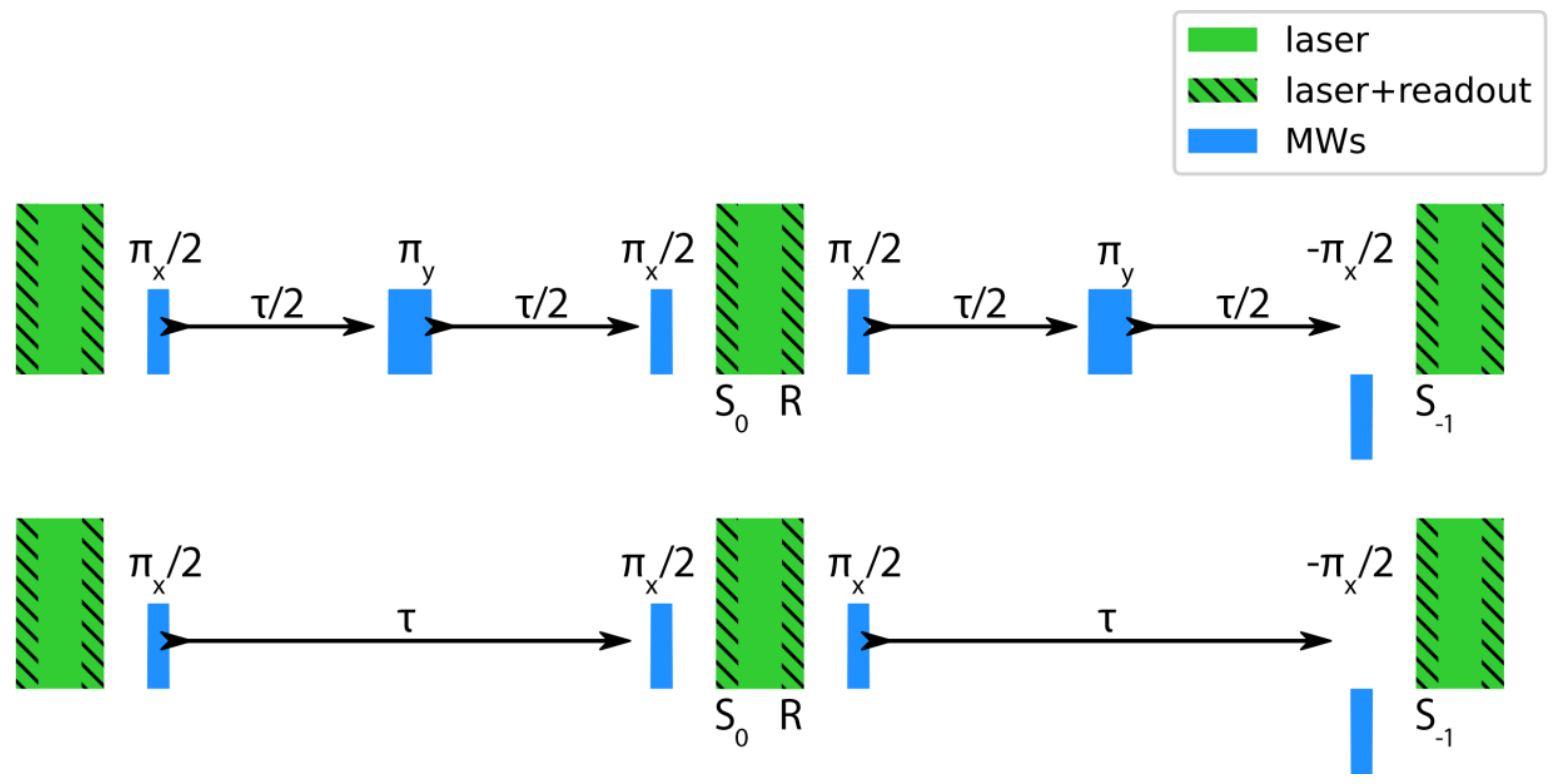

Figure S8: Coherence measurement sequences. Both the spin echo (a) and free induction decay (b) sequences have readout, initialization, state preparation, and state projection pulses. The spin echo sequence has a central $\pi$-pulse to refocus the spin. 

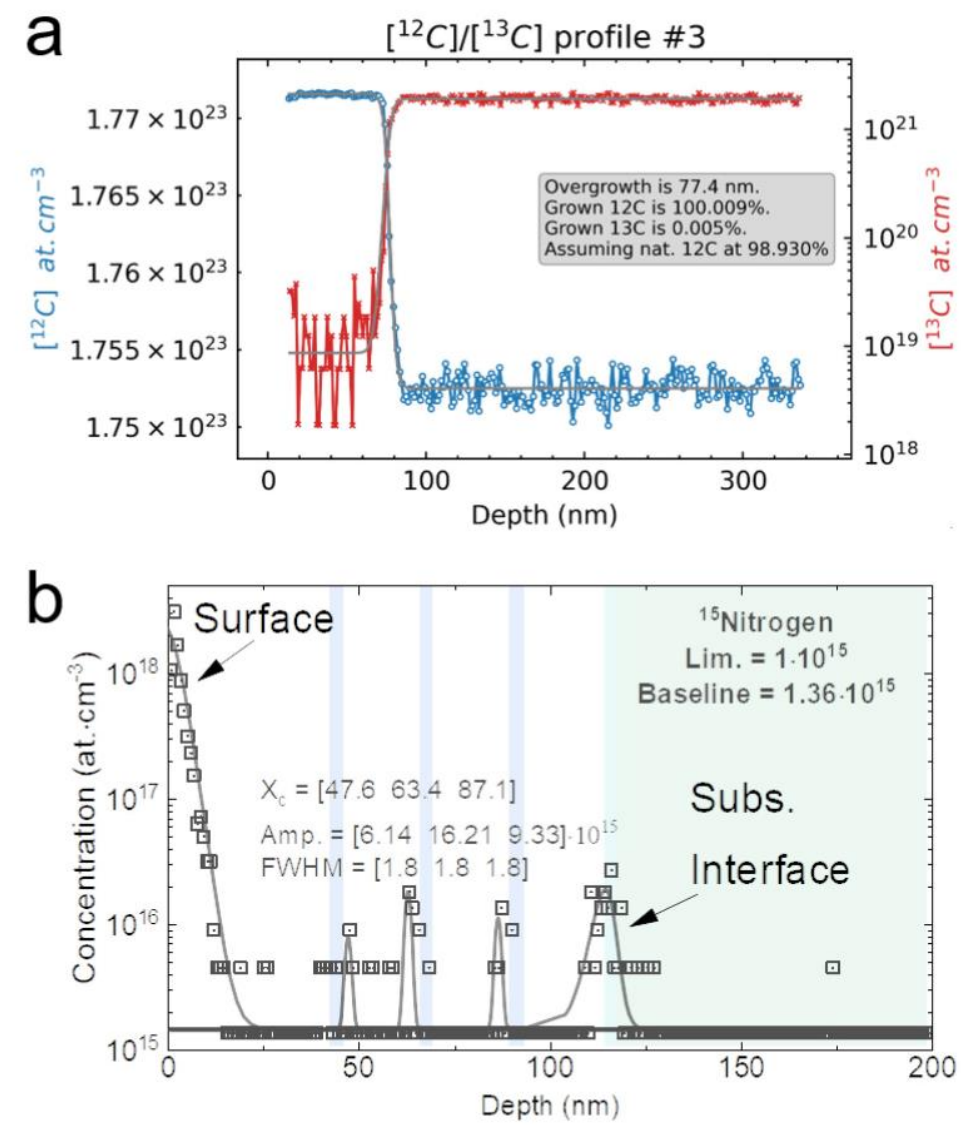

Figure S9: SIMS characterizations. (a) SIMS characterization of a typical isotopically enriched diamond growth using our standard overgrowth showing 99.99at. $\%{ }^{12} \mathrm{C}$ diamond. (b) Nitrogen doping effectiveness ${ }^{15} \mathrm{~N}$ (background contamination ${ }^{14} \mathrm{~N}$ not shown but done parallel to this) for a triple delta-doped overgrowth sample. Fitting of the Gaussians was used to quantify the nitrogen density. The FWHM is used as an approximation of the delta-doping layer thickness, whereas the amplitude is used as an approximation of the atomic density $\left(\mathrm{cm}^{-3}\right)$ within that layer. The detection limit is shown in graph. We also note a significant amount of nitrogen at the substrate/overgrowth and surface interfaces with ratios consistent of natural abundance of ${ }^{15} \mathrm{~N}$ in atmospheric nitrogen (measured via peak area ratio). 